\title{
Majorana Zero Modes Protected by Hopf Invariant in Topologically Trivial Superconductors
}

\author{
Zhongbo Yan, ${ }^{1}$ Ren $\mathrm{Bi}^{1}{ }^{1}$ and Zhong Wang ${ }^{1,2, *}$ \\ ${ }^{1}$ Institute for Advanced Study, Tsinghua University, Beijing, 100084, China \\ ${ }^{2}$ Collaborative Innovation Center of Quantum Matter, Beijing, 100871, China
}

\begin{abstract}
Majorana zero modes are usually attributed to topological superconductors. We study a class of twodimensional topologically trivial superconductors without chiral edge modes, which nevertheless host robust Majorana zero modes in topological defects. The construction of this minimal single-band model is facilitated by the Hopf map and the Hopf invariant. This work will stimulate investigations of Majorana zero modes in superconductors in the topologically trivial regime.
\end{abstract}

Majorana zero modes (MZMs) or Majorana bound states are exotic excitations predicted to exist in the vortex cores[1, 2] of two-dimensional (2D) topological superconductors [3-7] and at the ends of 1D topological superconductors[8]. Spatially separated MZMs give rise to degenerate ground states, which encode qubits immune to local dechoerence [8, 9]. Furthermore, unitary transformations among the ground states can be implemented by braiding [10-14] or measurements 15 , 16] of these modes, indicating that such qubits may become building blocks in topological quantum computation and information[17-22]. Therefore, MZMs have been vigorously pursued in condensed matter physics [23-29].

There have been a great variety of proposals for topological superconductors, including 2D semiconductor heterostructures [30, 31], topological insulatorsuperconductor proximity [32-36], 1D spin-orbit-coupled quantum wires[37-45], spiral magnetic chains on superconductors [46-50], Shockley mechanism[51, 52], and cold atom systems in 2D[53-56] and 1D[57, 58], etc. Experimentally, suggestive signatures of MZMs in both $1 \mathrm{D}[59-69]$ and 2D[70-75] topological superconductors have been found.

It is often implicitly assumed that topological superconductivity is a prerequisite for MZMs, accordingly, the chiral edge states go hand in hand with the vortex zero modes in 2D superconductors. In this Letter we show that certain topological defects [76-81] in 2D topologically trivial superconductors can support robust MZMs. Somewhat surprisingly, single-band superconductors suffice this purpose. The model Hamiltonian is related to the Hopf maps, which originally refer to nontrivial mappings from a 3D sphere $S^{3}$ to a 2D sphere $S^{2}$, characterized by the integer Hopf invariant 82, 83]. Mappings from a 3D torus $T^{3}$ to $S^{2}$ inherit the nontrivial topology from the mappings $S^{3} \rightarrow S^{2}$. The Hopf invariant has found interesting applications in nonlinear $\sigma$ models and spin systems [82, 84], Hopf insulators [85-90], liquid crystals [91], and quench dynamics of Chern insulators 92, 93].

Our model describes topologically trivial superconductors with zero Chern number and no chiral edge state. Nevertheless, a topological point defect is characterized by a Hopf invariant defined in the $\left(k_{x}, k_{y}, \theta\right)$ space, where $k_{x}, k_{y}$ are crystal momenta and $\theta$ is the polar angle[94](Fig[1 $)$. The parity (even/odd) of Hopf invariant determines the presence (absence) of robust MZMs, though the superconductor for ev-
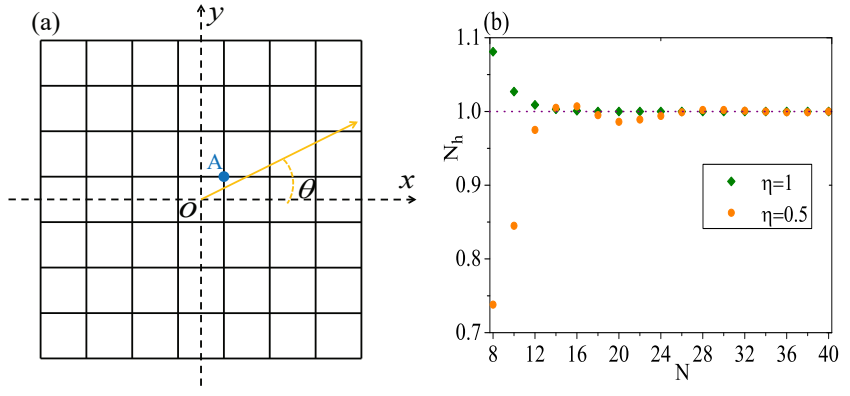

FIG. 1. (a) Sketch. The Hamiltonian varies as a function of $\theta$, creating a defect at $O=\left(x_{0}, y_{0}\right)$. In the polar coordinate, $\theta \equiv$ $\arctan \left(y-y_{0}\right) /\left(x-x_{0}\right)$. (b) The Hopf invariant for $\eta=1$ and $\eta=0.5$ $(n=1)$ in discretized-zone calculation, with $N^{3}$ grid points in the $(\mathbf{k}, \theta)$ space. As the grid becomes finer, $N_{h}$ converges rapidly to 1.

ery fixed $\theta$ is topologically trivial. Stimulated by this mechanism, which significantly differs from the magnetic-vortex origin of zero mode in topological $p$-wave superconductor [1, 2], we design trivial-superconductor-based (and vortex-free) Tjunctions harboring MZMs.

Zero modes.-Before studying topological defects, we consider spatially uniform 2D single-band Bogoliubov-de Gennes (BdG) Hamiltonians parameterized by $\lambda$ :

$$
H(\mathbf{k}, \lambda)=\left(\begin{array}{cc}
\xi_{\mathbf{k}}(\lambda) & \Delta_{\mathbf{k}}(\lambda) \\
\Delta_{\mathbf{k}}^{*}(\lambda) & -\xi_{-\mathbf{k}}(\lambda)
\end{array}\right)
$$

where $\mathbf{k}=\left(k_{x}, k_{y}\right), \xi_{\mathbf{k}}=E_{\mathbf{k}}-\mu, E_{\mathbf{k}}$ and $\mu$ is the energy and chemical potential, respectively, and $\Delta_{\mathbf{k}}$ is the Cooper pairing. It describes single-band spinless (or spin-fully-polarized) superconductors. This Hamiltonian can be written in terms of the Pauli matrices $\tau_{i}$ as

$$
H(\mathbf{k}, \lambda)=\sum_{i=x, y, z} d_{i}(\mathbf{k}, \lambda) \tau_{i}
$$

with $d_{x}=\operatorname{Re} \Delta_{\mathbf{k}}, d_{y}=-\operatorname{Im} \Delta_{\mathbf{k}}, d_{z}=\xi_{\mathbf{k}}$ (we have $\xi_{\mathbf{k}}=\xi_{-\mathbf{k}}$ in our model). For reason to become clear shortly, we take

$$
d_{i}=z^{\dagger} \tau_{i} z
$$

where $z=\left(z_{1}, z_{2}\right)^{T}$ and

$$
\begin{aligned}
& z_{1}=\sin k_{x}+i \sin k_{y}, \\
& z_{2}=\sin \lambda+i\left(\cos k_{x}+\cos k_{y}+\cos \lambda-m_{0}\right),
\end{aligned}
$$


with $m_{0}=\frac{3}{2}$. We can check that $\Delta_{-\mathbf{k}}=-\Delta_{\mathbf{k}}$ and $\Delta_{\left(-k_{y}, k_{x}\right)}=$ $i \Delta_{\left(k_{x}, k_{y}\right)}$, thus the pairing is $p$-wave. Given Eq. (4), the pairing $\Delta_{\mathbf{k}}$ is of the same order as the hopping $\xi_{\mathbf{k}}$. To describe weaklypairing superconductors, one may consider

$$
H_{\eta}(\mathbf{k}, \lambda)=\eta\left(d_{x} \tau_{x}+d_{y} \tau_{y}\right)+d_{z} \tau_{z}
$$

with a small but nonzero $\eta$. Nevertheless, tuning the value of $\eta$ does not close the energy gap, hence it does not qualitatively change the results. Thus we will simply take $\eta=1$ below. The mathematical form of Eq. (4) has been introduced for 3D Hopf insulators $85-90$ ], with $\lambda$ replaced by the third momentum $k_{z}$. The physical system we will study is nevertheless not directly related to Hopf insulator.

The familiar Chern number [1, 33, 95] $C$ that characterizes 2D topological superconductors can be obtained by a straightforward numerical calculation, which yields $C(\lambda)=0$ for every $\lambda$.

A topological defect can be generated if the parameter $\lambda$ depends on spatial coordinates, in a manner that the configuration cannot be smoothly deformed to a spatially uniform one. Let us focus on the defects with $\lambda$ depending on the polar angle $\theta$ (Fig 11a) as

$$
\lambda=n \theta
$$

where $n$ is an integer. These configurations are topologically nontrivial due to a nonzero Hopf invariant, as we now explain. The unit vector $\hat{\mathbf{d}}(\mathbf{k}, \theta) \equiv \frac{1}{\sqrt{\eta^{2}\left(d_{x}^{2}+d_{y}^{2}\right)+d_{z}^{2}}}\left(\eta d_{x}, \eta d_{y}, d_{z}\right)$ maps the $3 \mathrm{D}$ torus $T^{3}\left(k_{x}, k_{y}, \theta\right.$ are defined modulo $\left.2 \pi\right)$ to the 2D unit sphere $S^{2}$. For nonzero $n$, the inverseimage circles of two points on $S^{2}$ are linked[96]. To quantify such linking, the Hopf invariant can be defined $[82$, 85]: $N_{h}=-\frac{1}{4 \pi^{2}} \int d \theta d^{2} k \epsilon^{\mu \nu \rho} a_{\mu} \partial_{\nu} a_{\rho}$, where the integrating range is the Brillouin zone for $\mathbf{k}$ and $[0,2 \pi]$ for $\theta, a_{\mu}=$ $-i\left\langle\psi(\mathbf{k}, \theta)\left|\partial_{\mu}\right| \psi(\mathbf{k}, \theta)\right\rangle$, with $\mu, v, \rho=k_{x}, k_{y}, \theta$, and $|\psi\rangle$ is the negative-energy eigenfunction of $H_{\eta}(\mathbf{k}, \theta)$. Alternatively, we can define $A_{\mu}=a_{\mu} / 2 \pi, j^{\mu}=\epsilon^{\mu v \rho} \partial_{\nu} A_{\rho}=(1 / 8 \pi) \epsilon^{\mu v \rho} \hat{\mathbf{d}} \cdot\left(\partial_{\nu} \hat{\mathbf{d}} \times\right.$ $\left.\partial_{\rho} \hat{\mathbf{d}}\right)$, then [82, 85]

$$
N_{h}=-\int d^{2} k d \theta \mathbf{j} \cdot \mathbf{A} .
$$

It can be calculated numerically by discretizing the Brillouin zone [96]. The numerical result for $n=1$ is shown in Fig[1b. More generally, we have $N_{h}=n$. We will call the topological defects defined by Eq. (6) as Hopf defects.

To obtain energy spectra, we Fourier-transform the BdG Hamiltonian to real-space lattice, then numerically solve the Hamiltonian[96]. For $n=1$, two zero modes are found (Fig 2a), one of which is sharply localized around the defect, the other is localized at the sample boundary. The profile of particle component $\left(\tau_{z}=1\right.$ component, denoted by $u$ ) and hole component ( $\tau_{z}=-1$ component, denoted by $v$ ) is shown in the main figure and the inset, respectively. It is apparent that the zero modes are equal-weight superpositions of particle and hole components, which is a feature of MZMs (inspection of the wavefunction confirms that $u=v^{*}$ ).
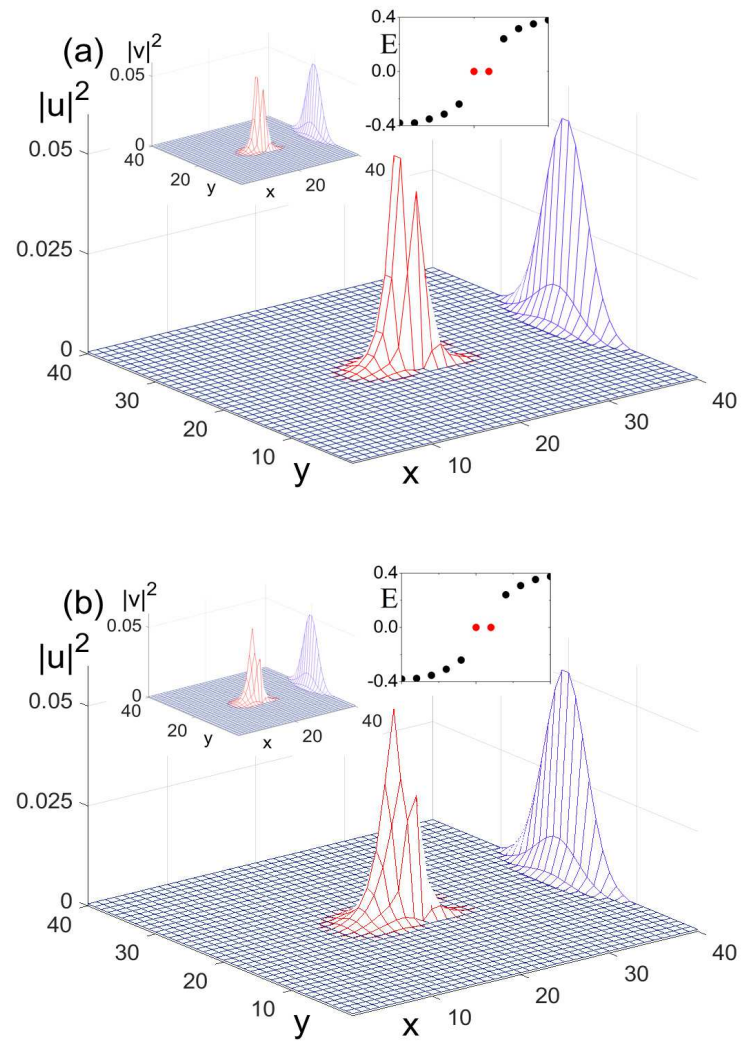

FIG. 2. (a) Profiles of two MZMs in a square sample with a Hopf defect $(n=1)$. The main figure shows the particle component $u$, and the left inset shows the hole component $v$. The right inset shows several energies close to 0 , with two zero energies colored in red. (b) is the same as (a) except that an impurity potential at the single site $A$ (indicated in Fig 19) is added.

To check the robustness of MZMs, we add an impurity potential $U c_{A}^{\dagger} c_{A}$ ( $c$ is the fermion operator) at a single site $A$ (specified in Fig 1 a), which amounts to adding a $U \tau_{z}$ term at site $A$ in the real-space BdG Hamiltonian. The numerical result for $U=1.0$ is shown in Fig $2 \mathrm{~b}$. The energies of MZMs remain pinned to $E=0$, though the mode profile is changed compared to Fig, 2 .

For $n=2$, we find two localized modes in the defect and two at the boundary (near $\theta=0$ and $\theta=\pi$ ). Unlike the $n=1$ case, the energies of defect modes are not pinned to zero. Higher $n$ 's are also calculated, and the results support the conclusion that there is a single robust MZM for odd-integer $n$, and no robust MZM for even-integer $n$, therefore, it is the $\mathrm{Z}_{2}$ Hopf invariant (even/odd) that determines the existence of MZM in the defect. One may notice that the Hopf invariant takes the form of a Chern-Simons invariant 76, 97], which is not accidental, because the latter is indeed a general topological invariant, nevertheless, it has been applied[76, 77] only to topologically nontrivial superconductors, for which it is just the product of the Chern number and the vorticity of pairing phase. Our model shows that nonzero Chern number is not a 

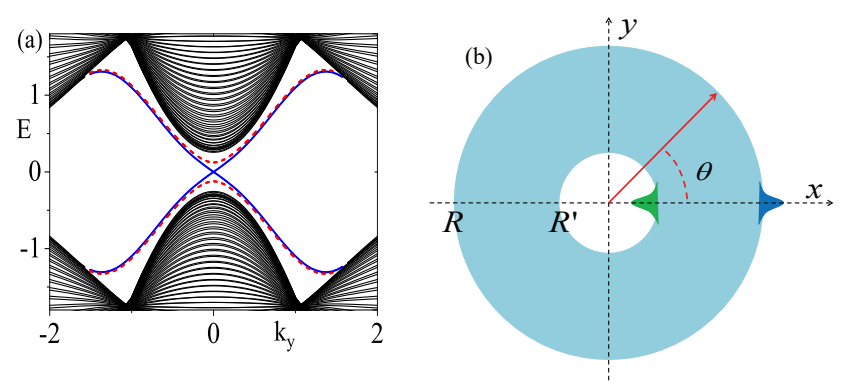

FIG. 3. (a) The energy bands for a ribbon with size $L_{x} \times L_{y}=$ $40 \times \infty ; \lambda=0$ (solid curves). The two dashed curves show the gapped edge modes for $\lambda=\pi / 20$ as a comparison. Each edge-mode band is doubly degenerate because a ribbon has two boundaries (ignoring a small splitting that exponentially decays as a function of $L_{x}$ ). (b) A large hollow disk with two MZMs illustrated. The inner MZM persists as the inner radius $R^{\prime} \rightarrow 0$, evolving to the defect MZM protected by Hopf invariant.

necessary condition for MZM.

Edge theory.-It is desirable to have an intuitive understanding of the MZM from the perspective of edge theory, which, as we will show, differs significantly from that of the chiral topological superconductor[1, 98, 99]. First, we numerically solved the edge states of open-boundary systems for various values of $\lambda$, and found that gapless edge modes exist only for $\lambda=0$. In Fig 3 a , we show the energy bands for a ribbon along $y$ direction. The gapless edge modes for $\lambda=0$ are shown as the solid blue lines. They are non-chiral, and are immediately gapped out when $\lambda$ is tuned away from 0 (edge modes of $\lambda=\pi / 20$ are shown in dashed curves), in other words, the edge modes are not topologically robust. This is consistent with the vanishing of Chern number.

This numerical observation is confirmed by analytic solutions. We consider a semi-infinite geometry with the sample occupying $x<0$ region, $k_{y}$ being a good quantum number. For $\lambda=0$, we obtain two degenerate edge modes at $k_{y}=0$, both of which are eigenfunctions of $\tau_{x}$ with eigenvalue $-1[96]$, thus they are equal-weight superpositions of particle and hole components. We introduce Pauli matrices $\sigma_{x, y, z}$ (unrelated to the $\tau_{x, y, z}$ matrices) in this two-dimensional space, so that the two eigenfunctions have $\sigma_{z}= \pm 1$, respectively. Including small $k_{y}$ and $\lambda$ as perturbations, we derive an effective theory[96]:

$$
H_{\mathrm{eff}}\left(k_{y}, \lambda\right)=-v k_{y} \sigma_{x}+M \lambda \sigma_{y},
$$

where the effective parameters $v, M$ are found to be both $3 / 4$ in our specific model[96]. Thus the edge-state spectra are $E_{ \pm}\left(k_{y}\right)= \pm \sqrt{v^{2} k_{y}^{2}+M^{2} \lambda^{2}}$. It is immediately clear that the edge states become gapped when $\lambda$ moves away from 0 , which is consistent with the numerical finding in Fig 3 a. As a comparison, we note that the edge spectrum of a chiral topological superconductor[1], $E\left(k_{y}\right)=v k_{y}$, cannot be gapped out.

Based on this effective edge theory, we proceed to study a hollow disk with polar-angle-dependent parameter, $\lambda=\theta$ (Fig 3 b). We are only concerned with low-energy modes, therefore, we focus on the neighborhood of $\theta=0$. Suppose that both the outer and inner radiuses $R, R^{\prime}$ are large. On the outer boundary, we have $\lambda=\theta=y / R$, thus the edge state spectra are given by solving $H_{\text {eff }}\left(k_{y} \rightarrow-i \partial_{y}, \lambda \rightarrow y / R\right) \psi=$ $E \psi$. More explicitly, it reads

$$
\left[i v \sigma_{x} \partial_{y}+(M / R) y \sigma_{y}\right] \psi=E \psi,
$$

which squares to $\left(-v^{2} \partial_{y}^{2}+M^{2} y^{2} / R^{2}-v M / R \sigma_{z}\right) \psi=E^{2} \psi$. This equation resembles the Schrödinger equation of harmonic oscillators, though $E$ is replaced by $E^{2}$, and there is a crucial additional $-v M / R \sigma_{z}$ term. The eigenfunctions are $\sigma_{z^{-}}$ eigenvectors (eigenvalues are denoted by $s_{z}= \pm 1$ ), with energies given by

$$
E_{\text {outer }}^{2}\left(s_{z}, n\right)=2(n+1 / 2) v M / R-s_{z} v M / R,
$$

where $n=0,1, \cdots$. There is a MZM in the $s_{z}=+1$ sector, with $n=0$, which is illustrated as the blue bump in Fig 3 b. Since this mode is the eigenfunction of $\tau_{x}$, it is an equalweight superposition of particle and hole components.

For a semi-infinite geometry with sample occupying the $x>0$ region, the effective edge theory is almost the same as Eq.(8), except that the sign of the first term reversed[96]. On the inner boundary of the hollow disk (again near $\theta=0$ ), we have $\lambda=\theta=y / R^{\prime}$, thus the edge-mode spectrum can be obtained from $\left[-i v \sigma_{x} \partial_{y}+\left(M / R^{\prime}\right) y \sigma_{y}\right] \psi=E \psi$, analogous to Eq. (9). The energies are given by

$$
E_{\text {inner }}^{2}\left(s_{z}, n\right)=2(n+1 / 2) v M / R^{\prime}+s_{z} v M / R^{\prime},
$$

which features a MZM in the $s_{z}=-1$ sector. The zero-mode wavefunction is

$$
\psi_{\text {inner }} \sim \exp \left(-M y^{2} / 2 v R^{\prime}\right)\left|s_{z}=-1\right\rangle,
$$

which is exponentially localized near $y=0$, namely $\theta=0$ (i1lustrated by the green bump in Fig $3 \mathrm{~b}$ ). All nonzero energies grow as $1 / R^{\prime}$ as $R^{\prime}$ is decreased, while the MZM remains at zero energy, evolving to the defect mode shown in Fig 2 . For a hollow disk with $\lambda=2 \theta$, there are two MZMs on the inner boundary for large $R^{\prime}$, near $\theta=0$ and $\theta=\pi$, respectively. Shrinking $R^{\prime}$ causes overlapping between them, which splits the two zero energies to nonzero values. This is consistent with the absence of MZM in the $n=2$ defect.

It is useful to compare our systems with the chiral topological superconductor, for which a magnetic vortex with $\pi$ flux hosts a MZM[1, 2, 32, 100]. In a hollow-disk geometry, this MZM comes from the chiral edge states on the boundary circle[1, 98, 99]. The MZM wavefunction is evenly distributed on the circle, which implies its sensitiveness to the magnetic flux. In contrast to this picture, the MZM in our model is not derived from chiral edge state, which is simply absent here, moreover, the MZM is exponentially localized near $\theta=0$ (Fig $3 \mathrm{~b})$, thus it is insensitive if a magnetic flux is inserted.

T-junctions.-So far, we have only studied configurations with $\lambda$ continuously varied. It is conceivable that the smooth 

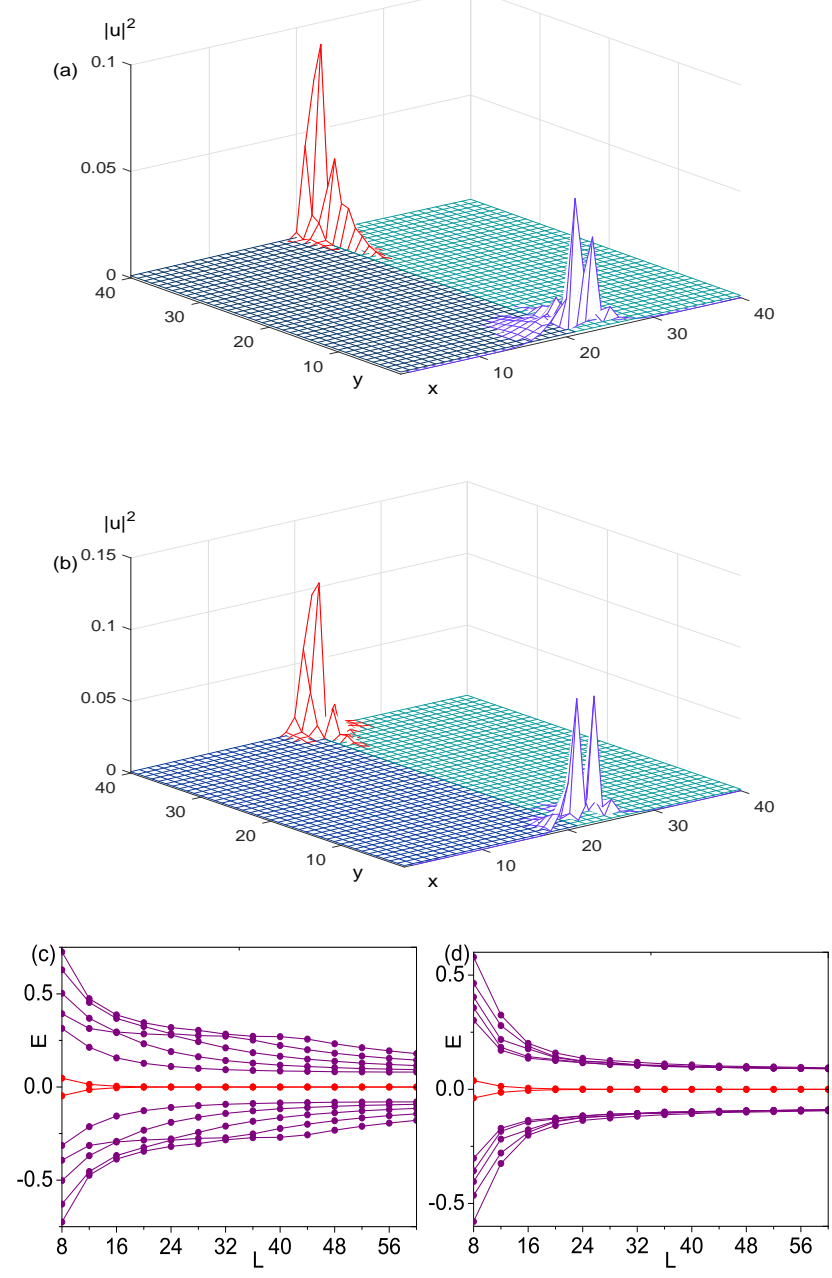

FIG. 4. (a) Superconductor-superconductor-vacuum T-junctions. The system size is $L^{2}$, with $L=40$. The $x>L / 2$ and $x \leq L / 2$ region, shown in different colors, is described by Eq.(2) with $\lambda$ taking $\lambda_{1}=0.1 \pi$ and $\lambda_{2}=\pi$, respectively. The peaks are the profiles of the two MZMs localized around the two T-junctions (each T-junction hosts one mode). (b) Superconductor-insulator-vacuum T-junctions. The parameters are the same as (a) except that $d_{x}$ and $d_{y}$ are tuned to 0 in the $\lambda_{2}$ region, so that $\lambda_{2}$ region is an insulator. (c) and (d) shows 12 energies closest to 0 , for system (a) and (b), respectively.

Hopf defect defined by Eq.(6) can be imitated by a discontinuous one, for instance, we may consider a T-junction:

$$
\lambda(\theta)= \begin{cases}\lambda_{1}, & \theta \in[0, \pi / 2] \\ \lambda_{2}, & \theta \in[\pi / 2, \pi] \\ \lambda_{3}, & \theta \in[\pi, 2 \pi] .\end{cases}
$$

$\lambda_{1,2,3}$ being three unequal constants. Such T-junctions will presumably be easier to realize than configurations with $\lambda$ smoothly varying in space.

We will study the simpler superconductor-superconductorvacuum T-junction by replacing the $\lambda_{3}$-region by the vacuum. Since the value of $\lambda$ in the vacuum is not well defined, this replacement is not fully justified in advance. Nevertheless, the numerical results thus obtained indicate that MZMs do exist in such T-junctions, as shown in Fig 4 h for $\lambda_{1}=0.1 \pi$, $\lambda_{2}=\pi$. There is certain arbitrariness in choosing the hopping at the boundary between the $\lambda_{1,2}$ regions, for which we keep only the nearest-neighbor hopping (discarding the nextnearest-neighbor hopping and the pairing) [101]. The energy eigenvalues near zero are shown in Fig 4 i (we show 12 of them), from which it is clear that the zero-mode levels are separate from all other energy levels by a finite gap in the $L \rightarrow \infty$ limit.

We have also studied superconductor-insulator-vacuum Tjunctions. To this end, we consider the $H_{\eta}$ in Eq.(5), in which taking $\eta=0$ amounts to removing the Cooper pairing. We notice that $H_{\eta=0}(\mathbf{k}, \lambda=\pi)$ describes an insulator without any Fermi surface [In contrast, $H_{\eta=0}(\mathbf{k}, \lambda=0.1 \pi)$ describes a metal]. Now we can design superconductor-insulator-vacuum T-junctions by taking $\eta=1$ in the $\lambda_{1}=0.1 \pi$ region, and $\eta=0$ in the $\lambda_{2}=\pi$ region. We find one MZM for each T-junction (shown in Fig 4b), and the energy gap between the zero-mode levels and other energy levels is apparent in Fig $4 \mathrm{~d}$. We emphasize that each region by itself is topologically trivial, in particular, the superconductor (with $\lambda=0.1 \pi$ ) is a topologically trivial one without gapless edge state.

Conclusions.-We have investigated the intriguing possibility of creating MZMs in 2D topologically trivial superconductors. The Hopf defect is constructed as a minimal model for this purpose. Furthermore, we studied the more accessible T-junctions constructed from topologically trivial superconductors. Hopefully, the trivial-superconductor-based approach will broaden the scope of searching MZMs in various superconductors. In particular, absence of chiral Majorana edge state in a 2D superconducting sample does not necessarily imply absence of robust MZM in its point defects.

We conclude with several remarks. First, we have focused on a single-band model, while many materials have multi-bands. We emphasize that the MZMs found here are nevertheless robust to small mixing with other bands, because a single localized MZM cannot move away from zero energy, as required by the intrinsic particle-hole symmetry of the BdG Hamiltonian[3]. Second, we have taken a simple model BdG Hamiltonians as our starting point (like Ref.[1]). More realistic Hamiltonians should be adopted when dealing with real materials, for instance, the semiconductorsuperconductor heterostructures [30, 31], for which our theory implies that robust MZMs can exist in certain defects (e.g. judiciously constructed T-junctions), without requiring the uniform system being tuned to the topologically nontrivial regime. This will be left for future works.

Acknowledgements.-We would like to thank Suk Bum Chung for helpful suggestions on the manuscript. This work is supported by NSFC (No. 11674189). Z.Y. is supported in part by China Postdoctoral Science Foundation (No. 2016M590082). 
*wangzhongemail@tsinghua.edu.cn

[1] N. Read and Dmitry Green, "Paired states of fermions in two dimensions with breaking of parity and timereversal symmetries and the fractional quantum hall effect," Phys. Rev. B 61, 10267-10297 (2000).

[2] GE Volovik, "Fermion zero modes on vortices in chiral superconductors," Journal of Experimental and Theoretical Physics Letters 70, 609-614 (1999).

[3] M. Z. Hasan and C. L. Kane, "Colloquium: Topological insulators," Rev. Mod. Phys. 82, 3045-3067 (2010)

[4] Xiao-Liang Qi and Shou-Cheng Zhang, "Topological insulators and superconductors," Rev. Mod. Phys. 83, 1057-1110 (2011)

[5] A. Bansil, Hsin Lin, and Tanmoy Das, "Colloquium : Topological band theory," Rev. Mod. Phys. 88, 021004 (2016)

[6] B Andrei Bernevig and Taylor L Hughes, Topological insulators and topological superconductors (Princeton University Press, 2013).

[7] Shun-Qing Shen, Topological Insulators: Dirac Equation in Condensed Matters, Vol. 174 (Springer Science \& Business Media, 2013).

[8] A Yu Kitaev, "Unpaired majorana fermions in quantum wires," Physics-Uspekhi 44, 131 (2001).

[9] Chetan Nayak, Steven H. Simon, Ady Stern, Michael Freedman, and Sankar Das Sarma, "Non-abelian anyons and topological quantum computation," Rev. Mod. Phys. 80, 1083 (2008).

[10] Gregory Moore and Nicholas Read, "Nonabelions in the fractional quantum hall effect," Nuclear Physics B 360, 362-396 (1991).

[11] Xiao-Gang Wen, "Non-abelian statistics in the fractional quantum hall states," Physical review letters 66, 802 (1991).

[12] D. A. Ivanov, "Non-abelian statistics of halfquantum vortices in p-wave superconductors," Phys. Rev. Lett. 86, 268-271 (2001)

[13] Chetan Nayak and Frank Wilczek, "2n-quasihole states realize $2 \mathrm{n}$-1-dimensional spinor braiding statistics in paired quantum hall states," Nuclear Physics B 479, 529-553 (1996).

[14] Sankar Das Sarma, Michael Freedman, and Chetan Nayak, "Topologically protected qubits from a possible non-abelian fractional quantum hall state," Phys. Rev. Lett. 94, 166802 (2005).

[15] S. Vijay and L. Fu, "Braiding without Braiding: Teleportation-Based Quantum Information Processing with Majorana Zero Modes," ArXiv e-prints (2016), arXiv:1609.00950 [cond-mat.mes-hall]

[16] Parsa Bonderson, Michael Freedman, and Chetan Nayak, "Measurement-only topological quantum computation," Phys. Rev. Lett. 101, 010501 (2008)

[17] David Aasen, Michael Hell, Ryan V. Mishmash, Andrew Higginbotham, Jeroen Danon, Martin Leijnse, Thomas S. Jespersen, Joshua A. Folk, Charles M. Marcus, Karsten Flensberg, and Jason Alicea, "Milestones toward majorana-based quantum computing,' Phys. Rev. X 6, 031016 (2016)

[18] Torsten Karzig, Yuval Oreg, Gil Refael, and Michael H. Freedman, "Universal geometric path to a robust majorana magic gate," Phys. Rev. X 6, 031019 (2016)

[19] T. Karzig, C. Knapp, R. Lutchyn, P. Bonderson, M. Hastings, C. Nayak, J. Alicea, K. Flensberg, S. Plugge, Y. Oreg, C. Marcus, and M. H. Freedman, "Scalable Designs for Quasiparticle-Poisoning-Protected Topological Quantum
Computation with Majorana Zero Modes," ArXiv e-prints (2016), arXiv:1610.05289 [cond-mat.mes-hall]

[20] B Van Heck, AR Akhmerov, F Hassler, M Burrello, and CWJ Beenakker, "Coulomb-assisted braiding of majorana fermions in a josephson junction array," New Journal of Physics 14, 035019 (2012).

[21] L. A. Landau, S. Plugge, E. Sela, A. Altland, S. M. Albrecht, and R. Egger, "Towards realistic implementations of a majorana surface code," Phys. Rev. Lett. 116, 050501 (2016)

[22] Dong-Ling Deng and Lu-Ming Duan, "Fault-tolerant quantum random-number generator certified by majorana fermions," Phys. Rev. A 88, 012323 (2013)

[23] Jason Alicea, "New directions in the pursuit of majorana fermions in solid state systems," Reports on Progress in Physics 75, 076501 (2012).

[24] C. W. J. Beenakker, "Search for Majorana Fermions in "Superconductors," Annual Review of Condensed Matter Physics 4, 113-136 (2013)

[25] Tudor D Stanescu and Sumanta Tewari, "Majorana fermions in semiconductor nanowires: fundamentals, modeling, and experiment," Journal of Physics: Condensed Matter 25, 233201 (2013).

[26] Martin Leijnse and Karsten Flensberg, "Introduction to topological superconductivity and majorana fermions," Semiconductor Science and Technology 27, 124003 (2012).

[27] Steven R. Elliott and Marcel Franz, "Colloquium : Majorana fermions in nuclear, particle, and solid-state physics," Rev. Mod. Phys. 87, 137-163 (2015)

[28] Sankar Das Sarma, Michael Freedman, and Chetan Nayak, "Majorana zero modes and topological quantum computation,” npj Quantum Information 1, 15001 (2015).

[29] Masatoshi Sato and Satoshi Fujimoto, "Majorana fermions and topology in superconductors," Journal of the Physical Society of Japan 85, 072001 (2016).

[30] Jay D. Sau, Roman M. Lutchyn, Sumanta Tewari, and S. Das Sarma, "Generic new platform for topological quantum computation using semiconductor heterostructures," Phys. Rev. Lett. 104, 040502 (2010).

[31] Jason Alicea, "Majorana fermions in a tunable semiconductor device," Phys. Rev. B 81, 125318 (2010).

[32] Liang Fu and C. L. Kane, "Superconducting proximity effect and majorana fermions at the surface of a topological insulator,' Phys. Rev. Lett. 100, 096407 (2008)

[33] Xiao-Liang Qi, Taylor L. Hughes, and Shou-Cheng Zhang, "Chiral topological superconductor from the quantum hall state," Phys. Rev. B 82, 184516 (2010)

[34] Suk Bum Chung, Xiao-Liang Qi, Joseph Maciejko, and ShouCheng Zhang, "Conductance and noise signatures of majorana backscattering," Phys. Rev. B 83, 100512 (2011)

[35] K. T. Law, Patrick A. Lee, and T. K. Ng, "Majorana fermion induced resonant andreev reflection," Phys. Rev. Lett. 103, 237001 (2009)

[36] A. R. Akhmerov, Johan Nilsson, and C. W. J. Beenakker, "Electrically detected interferometry of majorana fermions in a topological insulator," Phys. Rev. Lett. 102, 216404 (2009)

[37] Yuval Oreg, Gil Refael, and Felix von Oppen, "Helical liquids and majorana bound states in quantum wires," Phys. Rev. Lett. 105, 177002 (2010)

[38] Roman M. Lutchyn, Jay D. Sau, and S. Das Sarma, "Majorana fermions and a topological phase transition in semiconductor-superconductor heterostructures," Phys. Rev. Lett. 105, 077001 (2010)

[39] Jason Alicea, Yuval Oreg, Gil Refael, Felix von Oppen, and Matthew PA Fisher, "Non-abelian statistics and topological 
quantum information processing in 1d wire networks," Nature Physics 7, 412-417 (2011).

[40] Roman M. Lutchyn, Tudor D. Stanescu, and S. Das Sarma, "Search for majorana fermions in multiband semiconducting nanowires,' Phys. Rev. Lett. 106, 127001 (2011).

[41] Tudor D. Stanescu, Roman M. Lutchyn, and S. Das Sarma, "Majorana fermions in semiconductor nanowires," Phys. Rev. B 84, 144522 (2011)

[42] Andrew C. Potter and Patrick A. Lee, "Multichannel generalization of kitaev's majorana end states and a practical route to realize them in thin films," Phys. Rev. Lett. 105, 227003 (2010).

[43] Diego Rainis, Luka Trifunovic, Jelena Klinovaja, and Daniel Loss, "Towards a realistic transport modeling in a superconducting nanowire with majorana fermions," Phys. Rev. B 87, 024515 (2013)

[44] Elsa Prada, Pablo San-Jose, and Ramón Aguado, "Transport spectroscopy of $n s$ nanowire junctions with majorana fermions," Phys. Rev. B 86, 180503 (2012).

[45] S. Das Sarma, Jay D. Sau, and Tudor D. Stanescu, "Splitting of the zero-bias conductance peak as smoking gun evidence for the existence of the majorana mode in a superconductorsemiconductor nanowire,' Phys. Rev. B 86, 220506 (2012)

[46] T.-P. Choy, J. M. Edge, A. R. Akhmerov, and C. W. J. Beenakker, "Majorana fermions emerging from magnetic nanoparticles on a superconductor without spin-orbit coupling,' Phys. Rev. B 84, 195442 (2011)

[47] Ivar Martin and Alberto F. Morpurgo, "Majorana fermions in superconducting helical magnets," Phys. Rev. B 85, 144505 (2012)

[48] S. Nadj-Perge, I. K. Drozdov, B. A. Bernevig, and Ali Yazdani, "Proposal for realizing majorana fermions in chains of magnetic atoms on a superconductor," Phys. Rev. B 88, 020407 (2013)

[49] Jelena Klinovaja, Peter Stano, Ali Yazdani, and Daniel Loss, "Topological superconductivity and majorana fermions in rkky systems," Phys. Rev. Lett. 111, 186805 (2013).

[50] M. M. Vazifeh and M. Franz, "Self-organized topological state with majorana fermions," Phys. Rev. Lett. 111, 206802 (2013).

[51] M. Wimmer, A. R. Akhmerov, M. V. Medvedyeva, J. Tworzydło, and C. W. J. Beenakker, "Majorana bound states without vortices in topological superconductors with electrostatic defects," Phys. Rev. Lett. 105, 046803 (2010).

[52] Dong-Ling Deng, Sheng-Tao Wang, Kai Sun, and LuMing Duan, "Proposal for observing non-abelian statistics of majorana-shockley fermions in an optical lattice," Phys. Rev. B 91, 094513 (2015)

[53] Masatoshi Sato, Yoshiro Takahashi, and Satoshi Fujimoto, "Non-abelian topological order in $s$-wave superfluids of ultracold fermionic atoms," Phys. Rev. Lett. 103, 020401 (2009)

[54] Chuanwei Zhang, Sumanta Tewari, Roman M. Lutchyn, and S. Das Sarma, “ $p_{x}+i p_{y}$ superfluid from $s$-wave interactions of fermionic cold atoms," Phys. Rev. Lett. 101, 160401 (2008)

[55] Sumanta Tewari, S Das Sarma, Chetan Nayak, Chuanwei Zhang, and P Zoller, "Quantum computation using vortices and majorana zero modes of a $\mathrm{p} x+\mathrm{i} p \mathrm{y}$ superfluid of fermionic cold atoms," Physical review letters 98, 010506 (2007).

[56] Xiong-Jun Liu, K. T. Law, and T. K. Ng, "Realization of $2 \mathrm{~d}$ spin-orbit interaction and exotic topological orders in cold atoms," Phys. Rev. Lett. 112, 086401 (2014)

[57] Liang Jiang, Takuya Kitagawa, Jason Alicea, A. R. Akhmerov, David Pekker, Gil Refael, J. Ignacio Cirac, Eugene Dem- ler, Mikhail D. Lukin, and Peter Zoller, "Majorana fermions in equilibrium and in driven cold-atom quantum wires," Phys. Rev. Lett. 106, 220402 (2011)

[58] Sebastian Diehl, Enrique Rico, Mikhail A Baranov, and Peter Zoller, "Topology by dissipation in atomic quantum wires," Nature Physics 7, 971-977 (2011).

[59] Vincent Mourik, Kun Zuo, Sergey M Frolov, SR Plissard, EPAM Bakkers, and LP Kouwenhoven, "Signatures of majorana fermions in hybrid superconductor-semiconductor nanowire devices," Science 336, 1003-1007 (2012).

[60] Stevan Nadj-Perge, Ilya K Drozdov, Jian Li, Hua Chen, Sangjun Jeon, Jungpil Seo, Allan H MacDonald, B Andrei Bernevig, and Ali Yazdani, "Observation of majorana fermions in ferromagnetic atomic chains on a superconductor," Science 346, 602-607 (2014).

[61] Leonid P Rokhinson, Xinyu Liu, and Jacek K Furdyna, "The fractional ac josephson effect in a semiconductorsuperconductor nanowire as a signature of majorana particles," Nature Physics 8, 795-799 (2012).

[62] MT Deng, CL Yu, GY Huang, Marcus Larsson, Philippe Caroff, and HQ Xu, "Anomalous zero-bias conductance peak in a nb-insb nanowire-nb hybrid device," Nano letters 12, 6414-6419 (2012).

[63] Anindya Das, Yuval Ronen, Yonatan Most, Yuval Oreg, Moty Heiblum, and Hadas Shtrikman, "Zero-bias peaks and splitting in an al-inas nanowire topological superconductor as a signature of majorana fermions," Nature Physics 8, 887-895 (2012).

[64] A. D. K. Finck, D. J. Van Harlingen, P. K. Mohseni, K. Jung, and X. Li, "Anomalous modulation of a zerobias peak in a hybrid nanowire-superconductor device," Phys. Rev. Lett. 110, 126406 (2013)

[65] HOH Churchill, V Fatemi, Kasper Grove-Rasmussen, MT Deng, Philippe Caroff, HQ Xu, and Charles M Marcus, "Superconductor-nanowire devices from tunneling to the multichannel regime: Zero-bias oscillations and magnetoconductance crossover,' Physical Review B 87, 241401 (2013).

[66] SM Albrecht, AP Higginbotham, M Madsen, F Kuemmeth, TS Jespersen, Jesper Nygård, P Krogstrup, and CM Marcus, "Exponential protection of zero modes in majorana islands," Nature 531, 206-209 (2016).

[67] MT Deng, S Vaitiekènas, EB Hansen, J Danon, M Leijnse, K Flensberg, J Nygård, P Krogstrup, and CM Marcus, "Majorana bound state in a coupled quantum-dot hybrid-nanowire system," Science 354, 1557-1562 (2016).

[68] Marcel Franz, "Majorana's wires," Nature nanotechnology 8, 149-152 (2013).

[69] R. Pawlak, M. Kisiel, J. Klinovaja, T. Meier, S. Kawai, T. Glatzel, D. Loss, and E. Meyer, "Probing atomic structure and Majorana wavefunctions in mono-atomic $\mathrm{Fe}$ chains on superconducting $\mathrm{Pb}$ surface," npj Quantum Mechanics 2, 16035 (2016) arXiv:1505.06078 [physics.atm-clus]

[70] Jin-Peng Xu, Mei-Xiao Wang, Zhi Long Liu, Jian-Feng Ge, Xiaojun Yang, Canhua Liu, Zhu An Xu, Dandan Guan, Chun Lei Gao, Dong Qian, Ying Liu, Qiang-Hua Wang, FuChun Zhang, Qi-Kun Xue, and Jin-Feng Jia, "Experimental detection of a majorana mode in the core of a magnetic vortex inside a topological insulator-superconductor $\mathrm{bi}_{2} \mathrm{te}_{3} / \mathrm{nbse}_{2}$ heterostructure,' Phys. Rev. Lett. 114, 017001 (2015).

[71] Hao-Hua Sun, Kai-Wen Zhang, Lun-Hui Hu, Chuang Li, Guan-Yong Wang, Hai-Yang Ma, Zhu-An Xu, Chun-Lei Gao, Dan-Dan Guan, Yao-Yi Li, Canhua Liu, Dong Qian, Yi Zhou, Liang Fu, Shao-Chun Li, Fu-Chun Zhang, and Jin-Feng 
Jia, "Majorana zero mode detected with spin selective andreev reflection in the vortex of a topological superconductor," Phys. Rev. Lett. 116, 257003 (2016).

[72] Y.-F. Lv, W.-L. Wang, Y.-M. Zhang, H. Ding, W. Li, L. Wang, K. He, C.-L. Song, X.-C. Ma, and Q.-K. Xue, "Experimental Observation of Topological Superconductivity and Majorana Zero Modes on beta-Bi2Pd Thin Films," ArXiv e-prints (2016), arXiv:1607.07551 [cond-mat.supr-con].

[73] Mei-Xiao Wang, Canhua Liu, Jin-Peng Xu, Fang Yang, Lin Miao, Meng-Yu Yao, CL Gao, Chenyi Shen, Xucun Ma, $\mathrm{X}$ Chen, et al., "The coexistence of superconductivity and topological order in the bi2se3 thin films," Science 336, 5255 (2012).

[74] ZF Wang, Huimin Zhang, Defa Liu, Chong Liu, Chenjia Tang, Canli Song, Yong Zhong, Junping Peng, Fangsen Li, Caina Nie, LiLi Wang, X. J. Zhou, Xucun Ma, Q. K. Xue, and Feng Liu, "Topological edge states in a high-temperature superconductor fese/srtio3 (001) film," Nature Materials 15, 968-973 (2016).

[75] Q. L. He, L. Pan, A. L. Stern, E. Burks, X. Che, G. Yin, J. Wang, B. Lian, Q. Zhou, E. S. Choi, K. Murata, X. Kou, T. Nie, Q. Shao, Y. Fan, S.-C. Zhang, K. Liu, J. Xia, and K. L. Wang, "Chiral Majorana edge state in a quantum anomalous Hall insulator-superconductor structure," ArXiv e-prints (2016), arXiv:1606.05712 [cond-mat.supr-con].

[76] J. C. Y. Teo and C. L. Kane, "Topological defects and gapless modes in insulators and superconductors," Phys. Rev. B 82, 115120 (2010).

[77] Ching-Kai Chiu, Jeffrey C. Y. Teo, Andreas P. Schnyder, and Shinsei Ryu, "Classification of topological quantum matter with symmetries," Rev. Mod. Phys. 88, 035005 (2016)

[78] Ken Shiozaki and Masatoshi Sato, "Topology of crystalline insulators and superconductors," Phys. Rev. B 90, 165114 (2014)

[79] Dung-Hai Lee, Guang-Ming Zhang, and Tao Xiang, "Edge solitons of topological insulators and fractionalized quasiparticles in two dimensions," Phys. Rev. Lett. 99, 196805-4 (2007).

[80] Xiao-Liang Qi, Taylor Hughes, and Shou-Cheng Zhang, "Fractional charge and quantized current in the quantum spin Hall state," Nature Physics 4, 273 (2008).

[81] Ying Ran, Yi Zhang, and Ashvin Vishwanath, "Onedimensional topologically protected modes in topological insulators with lattice dislocations," Nature Physics 5, 298-303 (2009).

[82] Frank Wilczek and A. Zee, "Linking numbers, spin, and statistics of solitons," Phys. Rev. Lett. 51, 2250-2252 (1983).

[83] Mikio Nakahara, Geometry, topology and physics (CRC Press, 2003).

[84] Eduardo Fradkin, Field theories of condensed matter physics (Cambridge University Press, 2013).

[85] Joel E Moore, Ying Ran, and Xiao-Gang Wen, "Topological surface states in three-dimensional magnetic insulators," Physical review letters 101, 186805 (2008).

[86] D.-L. Deng, S.-T. Wang, C. Shen, and L.-M. Duan, "Hopf insulators and their topologically protected surface states," Phys. Rev. B 88, 201105 (2013)

[87] D.-L. Deng, S.-T. Wang, and L.-M. Duan, "Systematic construction of tight-binding hamiltonians for topological insulators and superconductors," Phys. Rev. B 89, 075126 (2014)

[88] D.-L. Deng, S.-T. Wang, K. Sun, and L.-M. Duan, "Probe knots and Hopf insulators with ultracold atoms," ArXiv eprints (2016), arXiv:1612.01518 [cond-mat.mes-hall]

[89] Ricardo Kennedy, "Topological hopf-chern insulators and the hopf superconductor,' Phys. Rev. B 94, 035137 (2016)
[90] C. Liu, F. Vafa, and C. Xu, "Symmetry Protected Topological Hopf Insulator and its Generalizations," ArXiv e-prints (2016), arXiv:1612.04905 [cond-mat.str-el]

[91] Paul J. Ackerman and Ivan I. Smalyukh, "Diversity of knot solitons in liquid crystals manifested by linking of preimages in torons and hopfions,' Phys. Rev. X 7, 011006 (2017).

[92] C. Wang, P. Zhang, X. Chen, J. Yu, and H. Zhai, "Measuring Topological Number of a Chern-Insulator from Quench Dynamics," ArXiv e-prints (2016), arXiv:1611.03304 [cond-mat.quant-gas]

[93] N. Fläschner, D. Vogel, M. Tarnowski, B. S. Rem, D.S. Lühmann, M. Heyl, J. C. Budich, L. Mathey, K. Sengstock, and C. Weitenberg, "Observation of a dynamical topological phase transition," ArXiv e-prints (2016), arXiv:1608.05616 [cond-mat.quant-gas]

[94] Converting a momentum variable to an angle variable was adopted in Refs. [97].

[95] D. J. Thouless, M. Kohmoto, M. P. Nightingale, and M. den Nijs, "Quantized hall conductance in a two-dimensional periodic potential," Phys. Rev. Lett. 49, 405-408 (1982)

[96] Supplemental Material.

[97] Xiao-Liang Qi, Taylor Hughes, and Shou-Cheng Zhang, "Topological Field Theory of Time-Reversal Invariant Insulators," Phys. Rev. B 78, 195424 (2008).

[98] Paul Fendley, Matthew P. A. Fisher, and Chetan Nayak, "Edge states and tunneling of non-abelian quasiparticles in the $v=5 / 2$ quantum hall state and $p+i p$ superconductors," Phys. Rev. B 75, 045317 (2007)

[99] Michael Stone and Rahul Roy, "Edge modes, edge currents, and gauge invariance in $p_{x}+i p_{y}$ superfluids and superconductors," Phys. Rev. B 69, 184511 (2004).

[100] Sumanta Tewari, S. Das Sarma, and Dung-Hai Lee, "Index theorem for the zero modes of majorana fermion vortices in chiral $p$-wave superconductors," Phys. Rev. Lett. 99, 037001 (2007)

[101] Keeping only the nearest-neighbor hopping means that (in real space) $H_{\mathrm{rs}}=(1.5-\cos \lambda) \tau_{z}$ for the boundary sites $\mathbf{r}=(L / 2, y)$ and $\mathbf{s}=(L / 2+1, y)$, where we take $\lambda$ as $\lambda_{2}=\pi$ (Another choice can be $\lambda=\lambda_{1}$. The choice is not unique). 


\section{Supplemental Material}

\section{EXPLICIT EXPRESSIONS OF $d_{x, y, z}$}

In the main article, we have taken $d_{i}=z^{\dagger} \tau_{i} z$ with $z=$ $\left(z_{1}, z_{2}\right)^{T}$ and

$$
\begin{aligned}
& z_{1}=\sin k_{x}+i \sin k_{y}, \\
& z_{2}=\sin \lambda+i\left(\cos k_{x}+\cos k_{y}+\cos \lambda-m_{0}\right) .
\end{aligned}
$$

For general $m_{0}$, the explicit form of these $d_{i}$ 's read

$d_{x}=2 \sin k_{x} \sin \lambda+2 \sin k_{y}\left(\cos k_{x}+\cos k_{y}+\cos \lambda-m_{0}\right)$,

$d_{y}=-2 \sin k_{y} \sin \lambda+2 \sin k_{x}\left(\cos k_{x}+\cos k_{y}+\cos \lambda-m_{0}\right)$, $d_{z}=\sin ^{2} k_{x}+\sin ^{2} k_{y}-\sin ^{2} \lambda-\left(\cos k_{x}+\cos k_{y}+\cos \lambda-m_{0}\right)^{2}(15)$ the kinetic energy term

$$
\begin{aligned}
\hat{H}_{0}= & -\frac{1}{2} \sum_{x, y}\left\{\left[(2 \cos \lambda-3)\left(c_{x, y}^{\dagger} c_{x+1, y}+c_{x, y}^{\dagger} c_{x, y+1}\right)+\left(c_{x, y}^{\dagger} c_{x+2, y}+c_{x, y}^{\dagger} c_{x, y+2}\right)+\right.\right. \\
& \left.\left.\left(c_{x, y}^{\dagger} c_{x+1, y+1}+c_{x, y}^{\dagger} c_{x+1, y-1}\right)\right]+ \text { h.c. }\right\}-\sum_{x, y}\left(\frac{13}{4}-3 \cos \lambda\right) c_{x, y}^{\dagger} c_{x, y},
\end{aligned}
$$

and the pairing term

$$
\begin{aligned}
\hat{H}_{\Delta}= & \eta \sum_{x, y}\left\{\left[\left(\frac{3}{2}-\cos \lambda-i \sin \lambda\right) c_{x, y}^{\dagger} c_{x+1, y}^{\dagger}+\text { h.c. }\right]+\left[\left(\sin \lambda-i \cos \lambda+\frac{3}{2} i\right) c_{x, y}^{\dagger} c_{x, y+1}^{\dagger}+\text { h.c. }\right]\right. \\
& \left.-\frac{1}{2}\left[c_{x, y}^{\dagger} c_{x+2, y}^{\dagger}+i c_{x, y}^{\dagger} c_{x, y+2}^{\dagger}+\text { h.c. }\right]-\left[\frac{1+i}{2} c_{x, y}^{\dagger} c_{x+1, y+1}^{\dagger}+\frac{1-i}{2} c_{x, y}^{\dagger} c_{x+1, y-1}^{\dagger}+\text { h.c. }\right]\right\},
\end{aligned}
$$

where $x, y$ are real-space coordinates taking integer values. As explained in the main article, $\lambda$ is simply a parameter of the Hamiltonian. We can see that the pairing between the nearestneighbor sites $(x, y)$ and $(x+1, y)$ is

$$
\Delta_{(x, y) ;(x+1, y)}=\eta\left(\frac{3}{2}-\cos \lambda-i \sin \lambda\right)
$$

and the pairing between sites $(x, y)$ and $(x, y+1)$ is

$$
\Delta_{(x, y) ;(x, y+1)}=\eta\left(\sin \lambda-i \cos \lambda+\frac{3}{2} i\right)
$$

thus

$$
\Delta_{(x, y) ;(x, y+1)}=i \Delta_{(x, y) ;(x+1, y)},
$$

showing a $p$-wave character in real space. Since the Chern number is zero (for any value of parameter $\lambda$ ), the superconductor is topologically trivial, as we have explained in the main article. 


\section{EXPLICIT EXPRESSIONS OF BDG HAMILTONIAN IN THE REAL SPACE: WITH A DEFECT}

In the previous section, we have focused on uniform systems with spatially independent $\lambda$. As explained in the main article, a topological defect can be created if the Hamiltonian parameter $\lambda$ depends on the polar angle $\theta$, which is measured from the defect center $\left(x_{0}, y_{0}\right)$, i.e.

$$
\theta_{x, y}=\arctan \frac{y-y_{0}}{x-x_{0}},
$$

such that the configuration cannot be smoothly deformed to a uniform one. The spatial dependence of the parameter $\lambda$ in our topological defects is given by

$$
\lambda_{x, y}=n \theta_{x, y} .
$$

Before proceeding, it is useful to point out that, since the hopping terms and the pairing terms are defined on the links instead of sites, it is natural to take the middle point of the link to define $\theta$, for instance, $\cos \theta c_{x, y}^{\dagger} c_{x+1, y}$ is taken as $\cos \theta_{x+1 / 2, y} c_{x, y}^{\dagger} c_{x+1, y}$ with $\theta_{x+1 / 2, y}=\arctan \left[\left(y-y_{0}\right) /(x+1 / 2-\right.$ $\left.x_{0}\right)$ ]. It is also viable to take a different convention, say $\cos \theta_{x, y} c_{x, y}^{\dagger} c_{x+1, y}$, which does not affect the existence of Majorana zero mode, as verified in our numerical calculations. The reason is that $\left|\theta_{x, y}-\theta_{x+1 / 2, y}\right| \rightarrow 0$ as $\left(x-x_{0}\right)^{2}+\left(y-y_{0}\right)^{2} \rightarrow \infty$, thus different choices merely differ in the near-defect-core region. The topological character of the defect is fixed by the Hamiltonian far from the defect center, which is not affected by modifying the near-defect-core region.

With the above technical aspect explained, we are ready to write down the real-space BdG Hamiltonian in the presence of a defect:

$$
\begin{aligned}
\hat{H}_{0}= & -\frac{1}{2} \sum_{x y}\left\{\left[\left(2 \cos n \theta_{x+\frac{1}{2}, y}-3\right) c_{x, y}^{\dagger} c_{x+1, y}+\left(2 \cos n \theta_{x, y+\frac{1}{2}}-3\right) c_{x, y}^{\dagger} c_{x, y+1}+\left(c_{x, y}^{\dagger} c_{x+2, y}+c_{x, y}^{\dagger} c_{x, y+2}\right)+\right.\right. \\
& \left.\left.\left(c_{x, y}^{\dagger} c_{x+1, y+1}+c_{x, y}^{\dagger} c_{x+1, y-1}\right)\right]+ \text { h.c. }\right\}-\sum_{x y}\left(\frac{13}{4}-3 \cos n \theta_{x, y}\right) c_{x, y}^{\dagger} c_{x, y}, \\
\hat{H}_{\Delta}= & \eta \sum_{x y}\left\{\left[\left(\frac{3}{2}-\cos n \theta_{x+\frac{1}{2}, y}-i \sin n \theta_{x+\frac{1}{2}, y}\right) c_{x, y}^{\dagger} c_{x+1, y}^{\dagger}+\text { h.c. }\right]\right. \\
& +\left[\left(\sin n \theta_{x, y+\frac{1}{2}}-i \cos n \theta_{x, y+\frac{1}{2}}+\frac{3}{2} i\right) c_{x, y}^{\dagger} c_{x, y+1}^{\dagger}+\text { h.c. }\right] \\
& \left.-\frac{1}{2}\left[c_{x, y}^{\dagger} c_{x+2, y}^{\dagger}+i c_{x, y}^{\dagger} c_{x, y+2}^{\dagger}+\text { h.c. }\right]-\left[\frac{1+i}{2} c_{x, y}^{\dagger} c_{x+1, y+1}^{\dagger}+\frac{1-i}{2} c_{x, y}^{\dagger} c_{x+1, y-1}^{\dagger}+\text { h.c. }\right]\right\},
\end{aligned}
$$

which simply replace the parameter $\lambda$ in Eq.(18) and Eq.(19) by $n \theta$. the real-space BdG Hamiltonian becomes

$$
\hat{H}=\sum_{x, y ; x^{\prime}, y^{\prime}} \Psi^{\dagger}(x, y) H_{x, y ; x^{\prime}, y^{\prime}} \Psi\left(x^{\prime}, y^{\prime}\right),
$$

It is a conventional step to define $\Psi(x, y)=\left(c_{x, y}, c_{x, y}^{\dagger}\right)^{T}$, and with nonzero elements of $H_{x, y ; x^{\prime}, y^{\prime}}$ given by

$$
\begin{aligned}
& H_{x, y ; x, y}=\left(\begin{array}{cc}
-\frac{13}{4}+3 \cos n \theta_{x, y} & 0 \\
0 & \frac{13}{4}-3 \cos n \theta_{x, y}
\end{array}\right), \\
& H_{x, y ; x \pm 1, y}=\left(\begin{array}{cc}
\frac{3}{2}-\cos n \theta_{x \pm \frac{1}{2}, y} & \mp i \sin n \theta_{x \pm \frac{1}{2}, y} \mp \cos n \theta_{x \pm \frac{1}{2}, y} \pm \frac{3}{2} \\
\mp i \sin n \theta_{x \pm \frac{1}{2}, y} \pm \cos n \theta_{x \pm \frac{1}{2}, y} \mp \frac{3}{2} & -\frac{3}{2}+\cos n \theta_{x \pm \frac{1}{2}, y}
\end{array}\right), \\
& H_{x, y ; x, y \pm 1}=\left(\begin{array}{cc}
\frac{3}{2}-\cos n \theta_{x, y \pm \frac{1}{2}} & \mp i \cos n \theta_{x, y \pm \frac{1}{2}} \pm \frac{3}{2} i \pm \sin n \theta_{x, y \pm \frac{1}{2}} \\
\mp i \cos n \theta_{x, y \pm \frac{1}{2}} \pm \frac{3}{2} i \mp \sin n \theta_{x, y \pm \frac{1}{2}} & -\frac{3}{2}+\cos n \theta_{x, y \pm \frac{1}{2}}
\end{array}\right), \\
& H_{x, y ; x \pm 2, y}=\left(\begin{array}{cc}
-\frac{1}{2} & \mp \frac{1}{2} \\
\pm \frac{1}{2} & \frac{1}{2}
\end{array}\right), \quad H_{x, y ; x, y \pm 2}=\left(\begin{array}{cc}
-\frac{1}{2} & \mp \frac{1}{2} i \\
\mp \frac{1}{2} i & \frac{1}{2}
\end{array}\right), \\
& H_{x, y ; x \pm 1, y \pm 1}=\left(\begin{array}{cc}
-\frac{1}{2} & \mp \frac{1}{2} i \mp \frac{1}{2} \\
\mp \frac{1}{2} i \pm \frac{1}{2} & \frac{1}{2}
\end{array}\right), \quad H_{x, y ; x \pm 1, y \mp 1}=\left(\begin{array}{cc}
-\frac{1}{2} & \pm \frac{1}{2} i \mp \frac{1}{2} \\
\pm \frac{1}{2} i \pm \frac{1}{2} & \frac{1}{2}
\end{array}\right) \text {. }
\end{aligned}
$$




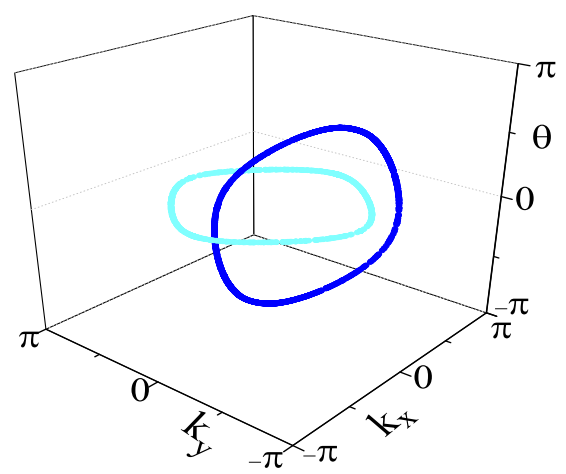

FIG. 5. The inverse images of $(0,0,1)$ (light cyan) and $(1,0,0)$ (dark blue) of the mapping $\hat{\mathbf{d}}(\mathbf{k}, \theta): T^{3} \rightarrow S^{2}$.

The rank of the matrix $H_{x, y ; x^{\prime}, y^{\prime}}$ is $2 L^{2}, L$ being the linear size of a square sample.

\section{NUMERICAL CALCULATION OF HOPF INVARIANT}

We start from the k-space BdG Hamiltonian:

$$
H_{\eta}(\mathbf{k}, \theta) \equiv \sum_{i} \tilde{d}_{i} \tau_{i}=\eta\left(d_{x} \tau_{x}+d_{y} \tau_{y}\right)+d_{z} \tau_{z}
$$

For $\eta \ll 1, H_{\eta}$ describes a superconductor with weak pairing (in the main article, we focused on the $\eta=1$ case).

As we explained in the main article, the unit vector

$$
\hat{\mathbf{d}}(\mathbf{k}, \theta) \equiv \frac{1}{\sqrt{\eta^{2}\left(d_{x}^{2}+d_{y}^{2}\right)+d_{z}^{2}}}\left(\eta d_{x}, \eta d_{y}, d_{z}\right)
$$

maps the 3D torus $T^{3}\left(k_{x}, k_{y}, \theta\right.$ are defined modulo $\left.2 \pi\right)$ to the two-dimensional unit sphere $S^{2}$. For nonzero $n$, the inverseimage circles of two points on $S^{2}$ are linked, which is illustrated in Fig 5 for the $n=1$ case.

The Hopf invariant, which characterizes the topological property of the Hamiltonian, is given by

$$
N_{h}=-\int d^{2} k d \theta \mathbf{j}(\mathbf{k}, \theta) \cdot \mathbf{A}(\mathbf{k}, \theta),
$$

where $\mathbf{j}(\mathbf{k}, \theta)=\left(j^{x}, j^{y}, j^{\theta}\right)$ (Note that the superscripts $x, y$ stand for $k_{x}, k_{y}$ ) takes the form of

$$
j^{\mu}=\frac{1}{8 \pi} \epsilon^{\mu \nu \rho} \hat{\mathbf{d}} \cdot\left(\partial_{\nu} \hat{\mathbf{d}} \times \partial_{\rho} \hat{\mathbf{d}}\right)
$$

and $\mathbf{A}=\left(A^{x}, A^{y}, A^{\theta}\right)$ (again, the superscripts $x, y$ stand for $\left.k_{x}, k_{y}\right)$ is the gauge potential satisfying $\nabla \times \mathbf{A}=\mathbf{j}$, where $\nabla=\left(\partial / \partial k_{x}, \partial / \partial k_{y}, \partial / \partial \theta\right)$. It is difficult to do the integration in Eq.(30) analytically. To do it numerically, we rewrite Eq.(30) into a discrete form:

$$
N_{h}=-\frac{(2 \pi)^{3}}{N^{3}} \sum_{\mathbf{k}, \theta} \mathbf{j}(\mathbf{k}, \theta) \cdot \mathbf{A}(\mathbf{k}, \theta),
$$

where $N$ is the number of lattice sites (the lattice constant is set to unit) and $k_{x, y}, \theta$ take discrete values in $\left\{-\pi,-\pi+\frac{2 \pi}{N}, \ldots, \pi-\right.$ $\left.\frac{2 \pi}{N}\right\}$.

To obtain the expression of $\mathbf{A}$ from $\mathbf{j}$, we do the following Fourier transformation:

$$
\begin{aligned}
\mathbf{A}(\mathbf{k}, \theta) & =\frac{1}{N^{3 / 2}} \sum_{\mathbf{q}} \mathbf{A}(\mathbf{q}) e^{-i\left(q_{x} k_{x}+q_{y} k_{y}+q_{\theta} \theta\right)}, \\
\mathbf{j}(\mathbf{k}, \theta) & =\frac{1}{N^{3 / 2}} \sum_{\mathbf{q}} \mathbf{j}(\mathbf{q}) e^{-i\left(q_{x} k_{x}+q_{y} k_{y}+q_{\theta} \theta\right)}
\end{aligned}
$$

where $\mathbf{q}=\left(q_{x}, q_{y}, q_{\theta}\right)$, whose components $q_{x, y, \theta}$ take discrete values in $\left\{-\frac{N}{2},-\frac{N}{2}+1, \ldots, \frac{N}{2}-1\right\}$. Under the gauge $\mathbf{q} \cdot \mathbf{A}=0$, it is readily found that

$$
\mathbf{A}(\mathbf{q})=-i \frac{\mathbf{q} \times \mathbf{j}(\mathbf{q})}{\mathbf{q}^{2}}
$$

thus the Hopf invariant becomes

$$
\begin{aligned}
N_{h} & =-\frac{(2 \pi)^{3}}{N^{3}} \sum_{\mathbf{q}} \mathbf{j}(-\mathbf{q}) \cdot \mathbf{A}(\mathbf{q}) \\
& =i \frac{(2 \pi)^{3}}{N^{3}} \sum_{\mathbf{q}} \frac{\mathbf{j}(-\mathbf{q}) \cdot(\mathbf{q} \times \mathbf{j}(\mathbf{q}))}{\mathbf{q}^{2}} .
\end{aligned}
$$

The numerical integration converges quite rapidly as we increase $N$. For $\lambda=n \theta$, we find $N_{h}=n$. The $n=1$ case is shown in Fig. 1b of the main article.

\section{MORE SUPPORTING DATA ON THE EFFECTS OF IMPURITY POTENTIAL}

In the main article, we have shown that the Majorana zero mode for $n=1$ is robust to an impurity potential, and remarked that robust Majorana zero mode is absent for eveninteger Hopf invariant. Here, more data on this is shown in Fig 6. As explained in the main article, an impurity potential $U c_{A}^{\dagger} c_{A}$ at a single site $A$ (indicated in Fig. 1a in the main article) is added to test the robustness of Majorana zero modes. For odd-integer Hopf invariant, Fig 6a shows that the impurity potential cannot move the zero mode energies away from $E=0$ (in consistent with the results given in the main article). For even-integer Hopf invariant, as shown in Fig $6 \mathrm{~b}$ and Fig 6, zero modes are generally absent in the defect in the presence of impurity potential (the blue lines in Fig 6 and Fig 6 are the energies for modes near the boundary, not near the defect at the system center). Note that several nonzero energy eigenvalues are almost independent of $U$ in Fig 6a, Fig 6 , and Fig 6 , , because their eigenfunctions are quite far from the impurity site $A$. 

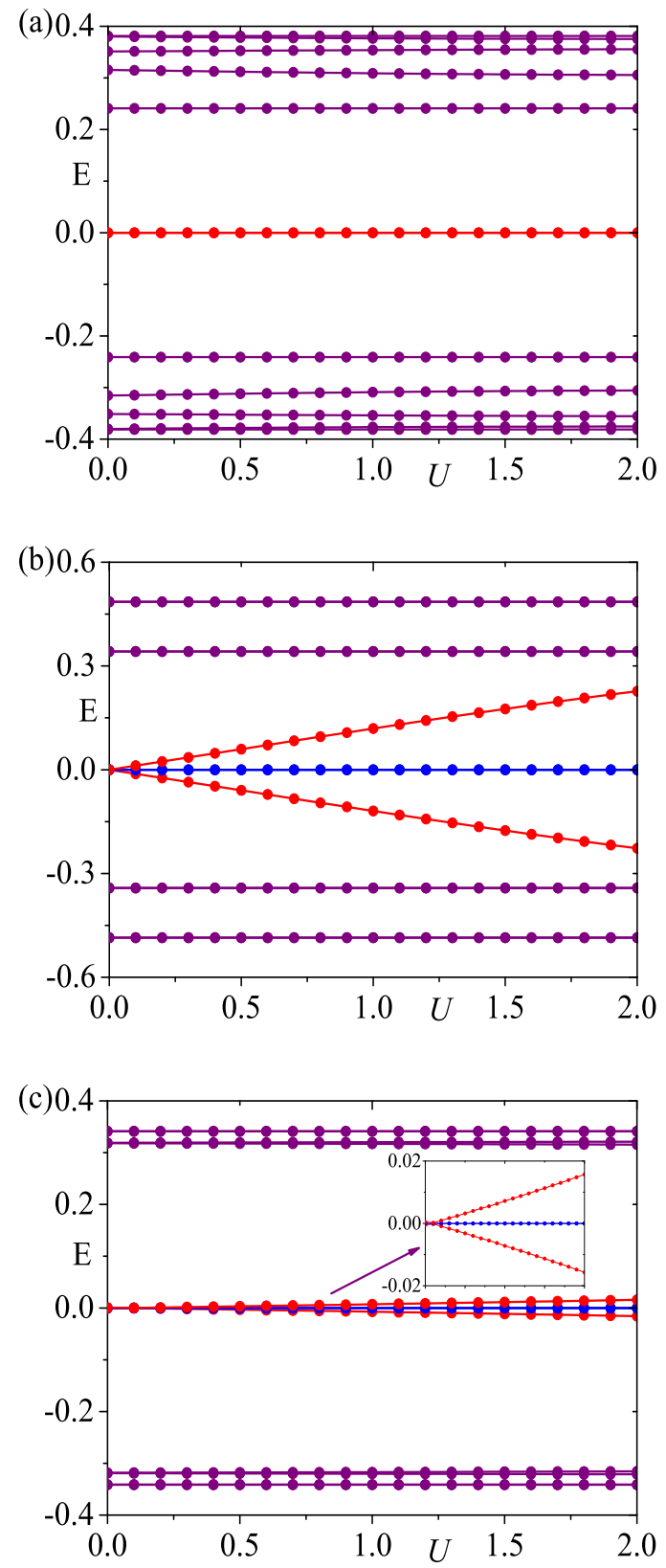

FIG. 6. The energy eigenvalues close to $E=0$ for three defects, as a function of an impurity potential $U$ at a single site $A$ (specified in Fig.1a in the main article). (a) $m_{0}=3 / 2$ and $n=1$, with Hopf invariant $N_{h}=1$. (b) $m_{0}=0$ and $n=1$, with $N_{h}=-2$. (c) $m_{0}=$ $3 / 2$, and $n=2$, with $N_{h}=2$. The inset of (c) provides a zoom-in view of the energy splitting. Several nonzero energy eigenvalues are almost independent of $U$ in (a), (b), and (c). The reason is that their eigenfunctions are quite far from the impurity site $A$.
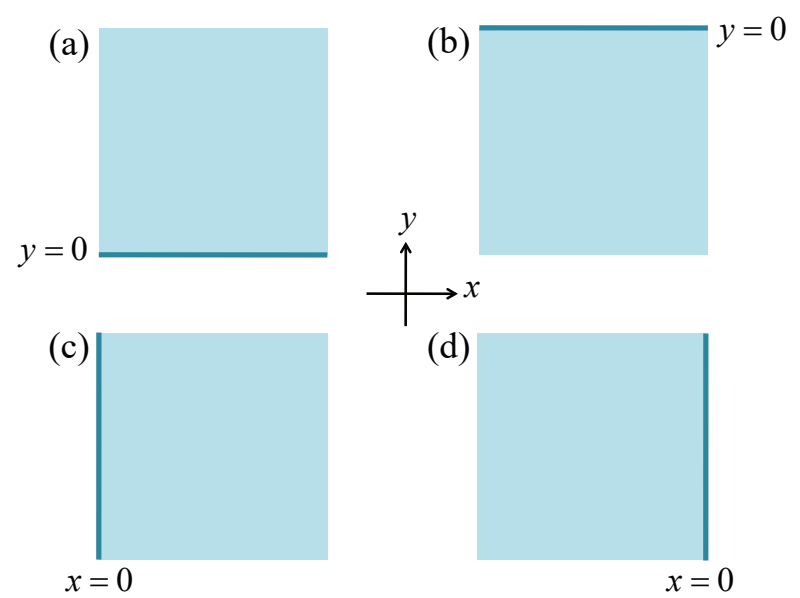

(d)

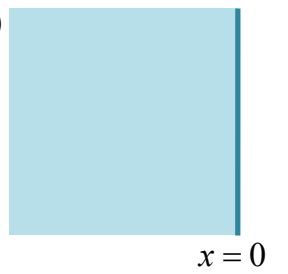

FIG. 7. Four geometries of the sample. The sample occupies (a) the $y>0$ region, (b) the $y<0$ region, (c) the $x>0$ region, (d) the $x<0$ region.

\section{EDGE THEORY FOR VARIOUS SAMPLE GEOMETRIES}

\section{Sample occupying $y>0$}

First, we study a semi-infinite geometry with the sample occupying the $y>0$ region (illustrated in Fig 7h). The momentum $k_{x}$ remains a good quantum number, while $k_{y}$ is not. In the $y$ direction, we use the real-space coordinate, which is an integer in our lattice model. The wave functions and energy eigenvalues can be obtained by solving the following equation

$$
\left(\begin{array}{ccccccc}
H_{0} & H_{1} & H_{2} & 0 & 0 & 0 & \cdots \\
H_{1}^{\dagger} & H_{0} & H_{1} & H_{2} & 0 & 0 & \cdots \\
H_{2}^{\dagger} & H_{1}^{\dagger} & H_{0} & H_{1} & H_{2} & 0 & \cdots \\
0 & H_{2}^{\dagger} & H_{1}^{\dagger} & H_{0} & H_{1} & H_{2} & \cdots \\
\vdots & \vdots & \vdots & \vdots & \vdots & \vdots & \ddots
\end{array}\right)\left(\begin{array}{c}
\psi_{1} \\
\psi_{2} \\
\psi_{3} \\
\psi_{4} \\
\vdots
\end{array}\right)=E\left(\begin{array}{c}
\psi_{1} \\
\psi_{2} \\
\psi_{3} \\
\psi_{4} \\
\vdots
\end{array}\right),
$$

where the subscript $j$ of $\psi_{j}$ is the $y$ coordinate, each $\psi_{j}$ is twocomponent, and 


$$
\begin{aligned}
& H_{0}\left(k_{x}, \lambda\right)=\left(\begin{array}{cc}
-\frac{13}{4}-\cos 2 k_{x}+3\left(\cos k_{x}+\cos \lambda\right)-2 \cos k_{x} \cos \lambda & 2 \sin k_{x} \sin \lambda-i\left(\sin 2 k_{x}+2 \sin k_{x} \cos \lambda-3 \sin k_{x}\right) \\
2 \sin k_{x} \sin \lambda+i\left(\sin 2 k_{x}+2 \sin k_{x} \cos \lambda-3 \sin k_{x}\right) & \frac{13}{4}+\cos 2 k_{x}-3\left(\cos k_{x}+\cos \lambda\right)+2 \cos k_{x} \cos \lambda
\end{array}\right), \\
& H_{1}\left(k_{x}, \lambda\right)=\left(\begin{array}{cc}
\frac{3}{2}-\cos k_{x}-\cos \lambda & -i\left(\cos k_{x}+\cos \lambda-\frac{3}{2}\right)-i \sin k_{x}+\sin \lambda \\
-i\left(\cos k_{x}+\cos \lambda-\frac{3}{2}\right)+i \sin k_{x}-\sin \lambda & -\frac{3}{2}+\cos k_{x}+\cos \lambda
\end{array}\right), \\
& H_{2}\left(k_{x}, \lambda\right)=\left(\begin{array}{cc}
-\frac{1}{2} & -\frac{1}{2} i \\
-\frac{1}{2} i & \frac{1}{2}
\end{array}\right) .
\end{aligned}
$$

Eq. (36) can be written compactly as $H\left(k_{x}, \lambda\right)|\Psi\rangle=E|\Psi\rangle, H$ denoting the large matrix at the left-hand-side of Eq. (36). Let us first find the zero-energy $(E=0)$ solutions, if any, at $k_{x}=0$ and $\lambda=0$. When $k_{x}=0$ and $\lambda=0, H_{i}$ 's simplify to

$$
\begin{aligned}
& H_{0}(0,0)=\left(\begin{array}{cc}
-\frac{1}{4} & 0 \\
0 & \frac{1}{4}
\end{array}\right), \\
& H_{1}(0,0)=H_{2}(0,0)=\left(\begin{array}{cc}
-\frac{1}{2} & -\frac{1}{2} i \\
-\frac{1}{2} i & \frac{1}{2}
\end{array}\right) .
\end{aligned}
$$

We can define $\tau_{ \pm}=\left(\tau_{z} \pm i \tau_{x}\right) / 2$, which is the raising/lowering operator of $\tau_{y}$, thus $H_{0}=-\left(\tau_{+}+\tau_{-}\right) / 4, H_{1}=H_{2}=-\tau_{+}$, and $H_{1}^{\dagger}=H_{2}^{\dagger}=-\tau_{-}$. Operating on the $\tau_{y}$ eigenvectors $\left|\chi_{ \pm}\right\rangle=$ $(1, \pm i)^{T} / \sqrt{2}$, they produce

$$
\begin{aligned}
& H_{1}\left|\chi_{+}\right\rangle=0, H_{1}^{\dagger}\left|\chi_{+}\right\rangle=-\left|\chi_{-}\right\rangle \\
& H_{1}\left|\chi_{-}\right\rangle=-\left\langle\chi_{+}\right\rangle, H_{1}^{\dagger}\left|\chi_{-}\right\rangle=0 ; \\
& H_{0}\left|\chi_{+}\right\rangle=-\frac{1}{4}\left|\chi_{-}\right\rangle, H_{0}\left|\chi_{-}\right\rangle=-\frac{1}{4}\left|\chi_{+}\right\rangle,
\end{aligned}
$$

We can check that the zero-energy wavefunctions take the form of

$$
|\Psi\rangle=\sum_{j} a_{j}|j\rangle \otimes\left|\chi_{-}\right\rangle
$$

where $|j\rangle$ is localized on the $j$-site:

$$
|j\rangle=(\underbrace{0,0, \ldots, 0}_{j-1}, 1,0, \ldots)^{T},
$$

with the coefficients $a_{j}$ satisfying the following iteration relation:

$$
a_{j}=-4\left(a_{j+1}+a_{j+2}\right) .
$$

We can see that $\psi_{j}=a_{j}\left|\chi_{-}\right\rangle$. It is straightforward to find two normalizable solutions for $a_{j}$ 's, which we will denote as $a_{j}=\alpha_{j}(j=1,2,3, \cdots)$ and $a_{j}=\beta_{j}(j=1,2,3, \cdots)$. The explicit expressions of $\alpha_{j}$ and $\beta_{j}$ are

$$
\alpha_{j}=C_{\alpha} \frac{(-1)^{j+1}}{2^{j-1}}
$$

and

$$
\beta_{j}=C_{\beta} \frac{(-1)^{j}(j-1)}{2^{j-2}},
$$

where $C_{\alpha}$ and $C_{\beta}$ are two normalization constants such that $\sum_{j}\left|\alpha_{j}\right|^{2}=1$ and $\sum_{j}\left|\beta_{j}\right|^{2}=1$. It is straightforward to find that $C_{\alpha}=\sqrt{3} / 2$ and $C_{\beta}=\sqrt{27 / 80}$.
We can check that the two wave functions $\left|\Psi_{1}\right\rangle=\sum_{j} \alpha_{j}|j\rangle \otimes$ $\left|\chi_{-}\right\rangle$and $\left|\Psi_{2}\right\rangle=\sum_{j} \beta_{j}|j\rangle \otimes\left|\chi_{-}\right\rangle$are not orthogonal. The orthogonalization can be achieved by the Gram-Schmidt orthogonalization. We have

$$
\begin{aligned}
& \left|\Psi_{1}^{\mathrm{o}}\right\rangle=\left|\Psi_{1}\right\rangle, \\
& \left|\Psi_{2}^{\mathrm{o}}\right\rangle=\mathcal{N}\left(\left|\Psi_{2}\right\rangle-\left|\Psi_{1}\right\rangle\left\langle\Psi_{1} \mid \Psi_{2}\right\rangle\right),
\end{aligned}
$$

where $\mathcal{N}=\sqrt{5} / 2$ is a normalization constant and the superscript "o" stands for "orthogonalization".

So far, we have focused on $k_{x}=0, \lambda=0$. In the neighbourhood of $k_{x}=0, \lambda=0$, we can expand the Hamiltonian $H$ to the first order of $k_{x}$ and $\lambda$, namely, $H_{i}\left(k_{x}, \lambda\right)=$ $H_{i}(0,0)+\Delta H_{i}\left(k_{x}, \lambda\right)$ with

$$
\begin{aligned}
\Delta H_{0}\left(k_{x}, \lambda\right) & =\left(\begin{array}{cc}
0 & -i k_{x} \\
i k_{x} & 0
\end{array}\right), \\
\Delta H_{1}\left(k_{x}, \lambda\right) & =\left(\begin{array}{cc}
0 & \lambda-i k_{x} \\
-\lambda+i k_{x} & 0
\end{array}\right) .
\end{aligned}
$$

It is readily seen that

$$
\begin{aligned}
& \left\langle\chi_{-}\left|\Delta H_{0}\right| \chi_{-}\right\rangle=-k_{x},\left\langle\chi_{-}\left|\Delta H_{1}\right| \chi_{-}\right\rangle=-\left(k_{x}+i \lambda\right), \\
& \left\langle\chi_{-}\left|\Delta H_{1}^{\dagger}\right| \chi_{-}\right\rangle=-\left(k_{x}-i \lambda\right) .
\end{aligned}
$$

Straightforward calculations lead to

$$
\begin{aligned}
\left\langle\Psi_{1}^{\mathrm{o}}|\Delta H| \Psi_{1}^{\mathrm{o}}\right\rangle & =-\left(\sum_{j=1} \alpha_{j}^{2}+2 \sum_{j=1} \alpha_{j} \alpha_{j+1}\right) k_{x} \\
& =-C_{\alpha}^{2}\left(\sum_{j=1} \frac{1}{2^{2 j-2}}-2 \sum_{j=1} \frac{1}{2^{2 j-1}}\right) k_{x} \\
& =0
\end{aligned}
$$


and

$$
\begin{aligned}
\left\langle\Psi_{2}^{\mathrm{o}}|\Delta H| \Psi_{2}^{\mathrm{o}}\right\rangle= & -\mathcal{N}^{2}\left[\sum_{j}\left[\beta_{j}-\left(\sum_{l} \alpha_{l} \beta_{l}\right) \alpha_{j}\right]^{2}+2 \sum_{j}\right. \\
& {\left.\left[\beta_{j}-\left(\sum_{l} \alpha_{l} \beta_{l}\right) \alpha_{j}\right]\left[\beta_{j+1}-\left(\sum_{l} \alpha_{l} \beta_{l}\right) \alpha_{j+1}\right]\right] k_{x} } \\
= & -\mathcal{N}^{2}\left[\left(\sum_{l} \alpha_{l} \beta_{l}\right)^{2}\left(\sum_{j=1} \alpha_{j}^{2}+2 \sum_{j=1} \alpha_{j} \alpha_{j+1}\right)\right. \\
& +\left(\sum_{j=1} \beta_{j}^{2}+2 \sum_{j=1} \beta_{j} \beta_{j+1}\right)-2\left(\sum_{l} \alpha_{l} \beta_{l}\right) \\
& \left.\times \sum_{j}\left(\alpha_{j} \beta_{j}+\alpha_{j} \beta_{j+1}+\alpha_{j+1} \beta_{j}\right)\right] k_{x} \\
= & -\mathcal{N}^{2}\left[\left(\sum_{j=1} \beta_{j}^{2}+2 \sum_{j=1} \beta_{j} \beta_{j+1}\right)-2\left(\sum_{l} \alpha_{l} \beta_{l}\right)\right. \\
& \left.\times \sum_{j}\left(\alpha_{j} \beta_{j}+\alpha_{j} \beta_{j+1}+\alpha_{j+1} \beta_{j}\right)\right] k_{x} \\
= & -\mathcal{N}^{2}\left[C_{\beta}^{2} \sum_{j=1} \frac{1-j}{2^{2 j-4}}-2\left(\sum_{l} \alpha_{l} \beta_{l}\right) C_{\alpha} C_{\beta} \sum_{j} \frac{1}{2^{2 j-2}}\right] k_{x} .
\end{aligned}
$$

Similarly, we find that

$$
\begin{aligned}
\left\langle\Psi_{1}^{\mathrm{o}}|\Delta H| \Psi_{2}^{\mathrm{o}}\right\rangle= & -\mathcal{N}\left[\sum _ { j } \left(\alpha_{j}\left[\beta_{j+1}-\left(\sum_{l} \alpha_{l} \beta_{l}\right) \alpha_{i+1}\right]\right.\right. \\
& \left.+\alpha_{j+1}\left[\beta_{j}-\left(\sum_{l} \alpha_{l} \beta_{l}\right) \alpha_{i}\right]\right) k_{x} \\
& +\sum_{j}\left(\alpha_{j}\left[\beta_{j+1}-\left(\sum_{l} \alpha_{l} \beta_{l}\right) \alpha_{i+1}\right]\right. \\
& \left.\left.-\alpha_{j+1}\left[\beta_{j}-\left(\sum_{l} \alpha_{l} \beta_{l}\right) \alpha_{i}\right]\right) i \lambda\right] \\
= & -\mathcal{N}\left[\left(\sum_{j}\left[\alpha_{j} \beta_{j+1}+\alpha_{j+1} \beta_{j}-2\left(\sum_{l} \alpha_{l} \beta_{l}\right) \alpha_{i} \alpha_{i+1}\right]\right) k_{x}\right. \\
& \left.+\sum_{j}\left(\alpha_{j} \beta_{j+1}-\alpha_{j+1} \beta_{j}\right) i \lambda\right] \\
= & -\mathcal{N}\left(C_{\alpha} C_{\beta} \sum_{j} \frac{1}{2^{2 j-2}}\right)\left(k_{x}+i \lambda\right) \\
= & -\frac{\mathcal{N} C_{\beta}}{C_{\alpha}}\left(k_{x}+i \lambda\right) \\
= & -\frac{3}{4}\left(k_{x}+i \lambda\right), \\
= & -\frac{3}{4}\left(k_{x}-i \lambda\right) . \\
\left\langle\Psi_{2}^{\mathrm{o}}|\Delta H| \Psi_{1}^{\mathrm{o}}\right\rangle= & \left\langle\Psi_{1}^{\mathrm{o}}|\Delta H| \Psi_{2}^{\mathrm{o}}\right\rangle^{*} \\
x_{x} &
\end{aligned}
$$

To summarize the above calculations, we have the following low-energy effective Hamiltonian for the edge states near $k_{x}=$ $0, \lambda=0$ :

Noting the mathematical identities

$$
\begin{aligned}
& \sum_{j} \alpha_{j} \beta_{j}=C_{\alpha} C_{\beta} \sum_{j} \frac{1-j}{2^{2 j-3}}, \\
& \sum_{j} \frac{1}{2^{2 j-2}}=\frac{1}{C_{\alpha}^{2}},
\end{aligned}
$$

we can see that

$$
\begin{aligned}
\left\langle\Psi_{2}^{\mathrm{o}}|\Delta H| \Psi_{2}^{\mathrm{o}}\right\rangle & =-\mathcal{N}^{2}\left[C_{\beta}^{2} \frac{2\left(\sum_{l} \alpha_{l} \beta_{l}\right)}{C_{\alpha} C_{\beta}}-2\left(\sum_{l} \alpha_{l} \beta_{l}\right) C_{\alpha} C_{\beta} \frac{1}{C_{\alpha}^{2}}\right] k_{x} \\
& =0 .
\end{aligned}
$$

$$
\begin{aligned}
H_{\mathrm{eff}} & =\left(\begin{array}{cc}
\left\langle\Psi_{1}^{\mathrm{o}}|\Delta H| \Psi_{1}^{\circ}\right\rangle & \left\langle\Psi_{1}^{\mathrm{o}}|\Delta H| \Psi_{2}^{\circ}\right\rangle \\
\left\langle\Psi_{2}^{\mathrm{o}}|\Delta H| \Psi_{1}^{\circ}\right\rangle & \left\langle\Psi_{2}^{\mathrm{o}}|\Delta H| \Psi_{2}^{\circ}\right\rangle
\end{array}\right) \\
& =-\frac{3}{4}\left(\begin{array}{cc}
0 & k_{x}+i \lambda \\
k_{x}-i \lambda & 0
\end{array}\right),
\end{aligned}
$$

or more compactly,

$$
H_{\mathrm{eff}}=\frac{3}{4}\left(-k_{x} \sigma_{x}+\lambda \sigma_{y}\right) .
$$

\section{Sample occupying $y<0$}

Now we study the sample occupying the $y<0$ region (Fig 7b), then the edge modes can be obtained by solving the following eigenvalue problem,

$$
\left(\begin{array}{ccccccc}
H_{0} & H_{1}^{\dagger} & H_{2}^{\dagger} & 0 & 0 & 0 & \cdots \\
H_{1} & H_{0} & H_{1}^{\dagger} & H_{2}^{\dagger} & 0 & 0 & \ldots \\
H_{2} & H_{1} & H_{0} & H_{1}^{\dagger} & H_{2}^{\dagger} & 0 & \cdots \\
0 & H_{2} & H_{1} & H_{0} & H_{1}^{\dagger} & H_{2}^{\dagger} & \cdots \\
\vdots & \vdots & \vdots & \vdots & \vdots & \vdots & \ddots
\end{array}\right)\left(\begin{array}{c}
\psi_{-1} \\
\psi_{-2} \\
\psi_{-3} \\
\psi_{-4} \\
\vdots
\end{array}\right)=E\left(\begin{array}{c}
\psi_{-1} \\
\psi_{-2} \\
\psi_{-3} \\
\psi_{-4} \\
\vdots
\end{array}\right) .(52)
$$

Following the same procedures in the previous section, we first find the solutions for $k_{x}=0$ and $\lambda=0$. We find two wave 
functions with $E=0$, one of which is $\left|\Psi_{1}^{\prime}\right\rangle=\sum_{j} \alpha_{j}|j\rangle \otimes\left|\chi_{+}\right\rangle$, and the other is $\left|\Psi_{2}^{\prime}\right\rangle=\sum_{j} \beta_{j}|j\rangle \otimes\left|\chi_{+}\right\rangle$. The orthogonalzation of the two wavefunctions are achieved by the Gram-Schmidt orthogonalization,

$$
\begin{aligned}
& \left|\Psi_{1}^{\prime o}\right\rangle=\left|\Psi_{1}^{\prime}\right\rangle, \\
& \left|\Psi_{2}^{\prime o}\right\rangle=\mathcal{N}\left(\left|\Psi_{2}^{\prime}\right\rangle-\left|\Psi_{1}^{\prime}\right\rangle\left\langle\Psi_{1}^{\prime} \mid \Psi_{2}^{\prime}\right\rangle\right) .
\end{aligned}
$$

It is readily checked that

$$
\begin{aligned}
& \left\langle\chi_{+}\left|\Delta H_{0}\right| \chi_{+}\right\rangle=k_{x},\left\langle\chi_{+}\left|\Delta H_{1}\right| \chi_{+}\right\rangle=k_{x}+i \lambda \\
& \left\langle\chi_{+}\left|\Delta H_{1}^{\dagger}\right| \chi_{+}\right\rangle=k_{x}-i \lambda
\end{aligned}
$$

Straightforward calculations yield

$$
\left(\begin{array}{cc}
\left\langle\Psi_{1}^{\prime \mathrm{o}}|\Delta H| \Psi_{1}^{\prime \mathrm{o}}\right\rangle & \left\langle\Psi_{1}^{\prime \mathrm{o}}|\Delta H| \Psi_{2}^{\prime \mathrm{o}}\right\rangle \\
\left\langle\Psi_{2}^{\prime \mathrm{o}}|\Delta H| \Psi_{1}^{\prime \mathrm{o}}\right\rangle & \left\langle\Psi_{2}^{\prime \mathrm{o}}|\Delta H| \Psi_{2}^{\prime \mathrm{o}}\right\rangle
\end{array}\right)=\frac{3}{4}\left(\begin{array}{cc}
0 & k_{x}-i \lambda \\
k_{x}+i \lambda & 0
\end{array}\right)
$$$$
\begin{aligned}
& \tilde{H}_{0}\left(k_{y}, \lambda\right)=\left(\begin{array}{cc}
-\frac{13}{4}-\cos 2 k_{y}+3\left(\cos k_{y}+\cos \lambda\right)-2 \cos k_{y} \cos \lambda & 2 i \sin k_{y} \sin \lambda+\left(\sin 2 k_{y}+2 \sin k_{y} \cos \lambda-3 \sin k_{y}\right) \\
-2 i \sin k_{y} \sin \lambda+\left(\sin 2 k_{y}+2 \sin k_{y} \cos \lambda-3 \sin k_{y}\right) & \frac{13}{4}+\cos 2 k_{y}-3\left(\cos k_{y}+\cos \lambda\right)+2 \cos k_{y} \cos \lambda
\end{array}\right) \\
& \tilde{H}_{1}\left(k_{y}, \lambda\right)=\left(\begin{array}{cc}
\frac{3}{2}-\cos k_{y}-\cos \lambda & -i \sin \lambda+\sin k_{y}-\left(\cos k_{y}+\cos \lambda-\frac{3}{2}\right) \\
-i \sin \lambda+\sin k_{y}+\left(\cos k_{y}+\cos \lambda-\frac{3}{2}\right) & -\frac{3}{2}+\cos k_{y}+\cos \lambda
\end{array}\right) \\
& \tilde{H}_{2}\left(k_{y}, \lambda\right)=\left(\begin{array}{cc}
-\frac{1}{2} & -\frac{1}{2} \\
\frac{1}{2} & \frac{1}{2}
\end{array}\right) .
\end{aligned}
$$

When $k_{y}=0$ and $\lambda=0$,

$$
\tilde{H}_{0}=\left(\begin{array}{cc}
-\frac{1}{4} & 0 \\
0 & \frac{1}{4}
\end{array}\right), \tilde{H}_{1}=\tilde{H}_{2}=\left(\begin{array}{cc}
-\frac{1}{2} & -\frac{1}{2} \\
\frac{1}{2} & \frac{1}{2}
\end{array}\right) .
$$

It is not difficult to see that

$$
\begin{aligned}
& \tilde{H}_{1}\left|\xi_{+}\right\rangle=0, \tilde{H}_{1}^{\dagger}\left|\xi_{+}\right\rangle=-\xi_{-}, \\
& \tilde{H}_{1}\left|\xi_{-}\right\rangle=-\left|\xi_{+}\right\rangle, \tilde{H}_{1}^{\dagger}\left|\xi_{-}\right\rangle=0, \\
& \tilde{H}_{0}\left|\xi_{+}\right\rangle=-\frac{1}{4}\left|\xi_{-}\right\rangle, \tilde{H}_{0}\left|\xi_{-}\right\rangle=-\frac{1}{4}\left|\xi_{+}\right\rangle,
\end{aligned}
$$

where $\left|\xi_{ \pm}\right\rangle=(1, \pm 1)^{T} / \sqrt{2}$ are the two eigenvectors of $\tau_{x}$. Following similar procedures as previous sections, we find two solutions at $E=0$ for $k_{y}=\lambda=0$. One is $\left|\tilde{\Psi}_{1}\right\rangle=$ $\sum_{j} \alpha_{j}|j\rangle \otimes\left|\xi_{+}\right\rangle$, and the other is $\left|\tilde{\Psi}_{2}\right\rangle=\sum_{j} \beta_{j}|j\rangle \otimes\left|\xi_{+}\right\rangle$. Again we adopt the Gram-Schmidt orthogonalization to define

$$
\begin{aligned}
& \left|\tilde{\Psi}_{1}^{\circ}\right\rangle=\left|\tilde{\Psi}_{1}\right\rangle, \\
& \left|\tilde{\Psi}_{2}^{\circ}\right\rangle=\mathcal{N}\left(\left|\tilde{\Psi}_{2}\right\rangle-\left|\tilde{\Psi}_{1}\right\rangle\left\langle\tilde{\Psi}_{1} \mid \tilde{\Psi}_{2}\right\rangle\right) .
\end{aligned}
$$

In the neighbourhood of $k_{y}=0$ and $\lambda=0, \tilde{H}_{i=0,1}$ can be expanded to the first order of $k_{y}$ and $\lambda$ :

$$
\tilde{H}_{i}\left(k_{y}, \lambda\right)=\tilde{H}_{i}(0,0)+\Delta \tilde{H}_{i}\left(k_{y}, \lambda\right)
$$

thus, the low-energy effective Hamiltonian for the edge state reads

$$
H_{\mathrm{eff}}=\frac{3}{4}\left(k_{x} \sigma_{x}+\lambda \sigma_{y}\right)
$$

\section{Sample occupying $x>0$}

For sample occupying the $x>0$ region (Fig 7 ), the edge modes can be obtained by solving the following eigenvalue problem,

$$
\left(\begin{array}{ccccccc}
\tilde{H}_{0} & \tilde{H}_{1} & \tilde{H}_{2} & 0 & 0 & 0 & \cdots \\
\tilde{H}_{1}^{\dagger} & \tilde{H}_{0} & \tilde{H}_{1} & \tilde{H}_{2} & 0 & 0 & \ldots \\
\tilde{H}_{2}^{\dagger} & \tilde{H}_{1}^{\dagger} & \tilde{H}_{0} & \tilde{H}_{1} & \tilde{H}_{2} & 0 & \ldots \\
0 & \tilde{H}_{2}^{\dagger} & \tilde{H}_{1}^{\dagger} & \tilde{H}_{0} & \tilde{H}_{1} & \tilde{H}_{2} & \cdots \\
\vdots & \vdots & \vdots & \vdots & \vdots & \vdots & \ddots
\end{array}\right)\left(\begin{array}{c}
\tilde{\psi}_{1} \\
\tilde{\psi}_{2} \\
\tilde{\psi}_{3} \\
\tilde{\psi}_{4} \\
\vdots
\end{array}\right)=E\left(\begin{array}{c}
\tilde{\psi}_{1} \\
\tilde{\psi}_{2} \\
\tilde{\psi}_{3} \\
\tilde{\psi}_{4} \\
\vdots
\end{array}\right),
$$

where 


\section{Sample occupying $x<0$}

Finally, we study the sample occupying the $x<0$ region (Fig,7d). We need to solve the eigenvalue equation:

$$
\left(\begin{array}{ccccccc}
\tilde{H}_{0} & \tilde{H}_{1}^{\dagger} & \tilde{H}_{2}^{\dagger} & 0 & 0 & 0 & \ldots \\
\tilde{H}_{1} & \tilde{H}_{0} & \tilde{H}_{1}^{\dagger} & \tilde{H}_{2}^{\dagger} & 0 & 0 & \ldots \\
\tilde{H}_{2} & \tilde{H}_{1} & \tilde{H}_{0} & \tilde{H}_{1}^{\dagger} & \tilde{H}_{2}^{\dagger} & 0 & \ldots \\
0 & \tilde{H}_{2} & \tilde{H}_{1} & \tilde{H}_{0} & \tilde{H}_{1} & \tilde{H}_{2}^{\dagger} & \ldots \\
\vdots & \vdots & \vdots & \vdots & \vdots & \vdots & \ddots
\end{array}\right)\left(\begin{array}{c}
\tilde{\psi}_{-1} \\
\tilde{\psi}_{-2} \\
\tilde{\psi}_{-3} \\
\tilde{\psi}_{-4} \\
\vdots
\end{array}\right)=E\left(\begin{array}{c}
\tilde{\psi}_{-1} \\
\tilde{\psi}_{-2} \\
\tilde{\psi}_{-3} \\
\tilde{\psi}_{-4} \\
\vdots
\end{array}\right) .(67)
$$

Following the same steps as in previous sections, we find two $E=0$ modes at $k_{y}=0$ and $\lambda=0$, one of which is $\left|\tilde{\Psi}_{1}^{\prime}\right\rangle=\sum_{j} \alpha_{j}|j\rangle \otimes\left|\xi_{-}\right\rangle$, and the other is $\left|\tilde{\Psi}_{2}^{\prime}\right\rangle=\sum_{j} \beta_{j}|j\rangle \otimes\left|\xi_{-}\right\rangle$, where we continue to use $\left|\xi_{ \pm}\right\rangle=(1, \pm 1)^{T} / \sqrt{2}$ to denote the two eigenvectors of $\tau_{x}$. After orthogonalzation, the wave functions take the form of

$$
\begin{aligned}
& \left|\tilde{\Psi}_{1}^{\prime}{ }^{o}\right\rangle=\left|\tilde{\Psi}_{1}^{\prime}\right\rangle, \\
& \left|\tilde{\Psi}_{2}^{\prime o}\right\rangle=\mathcal{N}\left(\left|\tilde{\Psi}_{2}^{\prime}\right\rangle-\left|\tilde{\Psi}_{1}^{\prime}\right\rangle\left\langle\tilde{\Psi}_{1}^{\prime} \mid \tilde{\Psi}_{2}^{\prime}\right\rangle\right) .
\end{aligned}
$$

In the neighborhood of $k_{y}=\lambda=0$, we expand the Hamiltonian to the first order of $k_{y}$ and $\lambda: \tilde{H}_{i}\left(k_{y}, \lambda\right)=\tilde{H}_{i}(0,0)+$ $\Delta \tilde{H}_{i}\left(k_{y}, \lambda\right)$, which satisfy

$$
\begin{aligned}
\left\langle\xi_{-}\left|\Delta \tilde{H}_{0}\right| \xi_{-}\right\rangle & =-k_{y},\left\langle\xi_{-}\left|\Delta \tilde{H}_{1}\right| \xi_{-}\right\rangle=-\left(k_{y}-i \lambda\right), \\
\left\langle\xi_{-}\left|\Delta \tilde{H}_{1}^{\dagger}\right| \xi_{-}\right\rangle & =-\left(k_{y}+i \lambda\right),
\end{aligned}
$$

therefore, we have

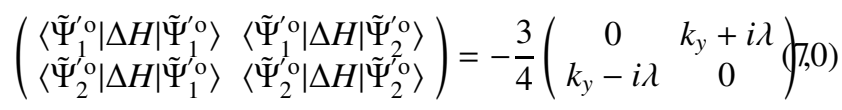

thus the low-energy effective Hamiltonian for the edge state is given by

$$
H_{\mathrm{eff}}=\frac{3}{4}\left(-k_{y} \sigma_{x}+\lambda \sigma_{y}\right)
$$

